OPEN ACCESS

Edited by: Lauriane Sollelis,

University of Glasgow,

United Kingdom

Reviewed by:

Qingfeng Zhang,

Tongji University, China

Michaela Petter,

University Hospital Erlangen, Germany

${ }^{*}$ Correspondence:

Till S. Voss

till.voss@swisstph.ch

Nicolas M. B. Brancucci

nicolas.brancucci@swisstph.ch

${ }^{t}$ These authors have contributed equally to this work and share last authorship

Specialty section: This article was submitted to

Parasite and Host, a section of the journal

Frontiers in Cellular and Infection Microbiology

Received: 26 October 2021 Accepted: 21 January 2022 Published: 11 February 2022

Citation:

Thommen BT, Passecker A, Buser T, Hitz E, Voss TS and Brancucci NMB (2022) Revisiting the Effect of

Pharmaceuticals on Transmission Stage Formation in the Malaria Parasite Plasmodium falciparum. Front. Cell. Infect. Microbiol. 12:802341. doi: 10.3389/fcimb.2022.802341

\section{Revisiting the Effect of Pharmaceuticals on Transmission Stage Formation in the Malaria Parasite Plasmodium falciparum}

\author{
Basil T. Thommen ${ }^{1,2}$, Armin Passecker ${ }^{1,2}$, Tamara Buser ${ }^{1,2}$, Eva Hitz ${ }^{1,2}$, Till S. Voss ${ }^{1,2 \star t}$ \\ and Nicolas M. B. Brancucci ${ }^{1,2 * T}$ \\ ${ }^{1}$ Department of Medical Parasitology and Infection Biology, Swiss Tropical and Public Health Institute, Allschwil, Switzerland, \\ 2 University of Basel, Basel, Switzerland
}

Malaria parasites rely on specialized stages, called gametocytes, to ensure human-tohuman transmission. The formation of these sexual precursor cells is initiated by commitment of blood stage parasites to the sexual differentiation pathway. Plasmodium falciparum, the most virulent of six parasite species infecting humans, employs nutrient sensing to control the rate at which sexual commitment is initiated, and the presence of stress-inducing factors, including antimalarial drugs, has been linked to increased gametocyte production in vitro and in vivo. These observations suggest that therapeutic interventions may promote gametocytogenesis and malaria transmission. Here, we engineered a $P$. falciparum reporter line to quantify sexual commitment rates after exposure to antimalarials and other pharmaceuticals commonly prescribed in malaria-endemic regions. Our data reveal that some of the tested drugs indeed have the capacity to elevate sexual commitment rates in vitro. Importantly, however, these effects are only observed at drug concentrations that inhibit parasite survival and only rarely result in a net increase of gametocyte production. Using a drug-resistant parasite reporter line, we further show that the gametocytogenesis-promoting effect of drugs is linked to general stress responses rather than to compound-specific activities. Altogether, we did not observe evidence for mechanistic links between the regulation of sexual commitment and the activity of commonly used pharmaceuticals in vitro. Our data hence does not support scenarios in which currently applied therapeutic interventions would promote the spread of drug-resistant parasites or malaria transmission in general.

Keywords: sexual commitment, Plasmodium falciparum, gametocytes, malaria transmission stages, malaria, antimalarials, high content imaging $(\mathrm{HCl})$ 


\section{INTRODUCTION}

Causing an estimated 200 million clinical cases and more than 400 ' 000 deaths annually, malaria represents one of the major threats to global public health (WHO, 2020). Malaria parasites resistant to current drug interventions, including the frontline artemisinin-based combination therapies (ACTs), are emerging and endanger malaria eradication campaigns (Ippolito et al., 2017). Among the six Plasmodium species infecting humans, $P$. falciparum is the most virulent and accounts for the majority of severe and lethal malaria cases (WHO, 2020). Following the injection of sporozoites by an infected Anopheles mosquito, parasites reproduce within hepatocytes before initiating the symptomatic phase of infection in the human blood. The latter is characterized by continuous rounds of erythrocyte invasion, asexual replication, host cell rupture, and the release of merozoites ready to invade new red blood cells (RBCs). During each of these 48-hour long intra-erythrocytic replication cycles, a small subset of parasites switches away from asexual replication and instead commits to sexual development, resulting in the formation of transmissible gametocytes (Josling et al., 2018). P. falciparum gametocytes sequester in deep tissue, including the bone marrow parenchyma, where they undergo a series of developmental steps (I-V) before re-entering the blood stream as mature and transmission-competent stage $\mathrm{V}$ gametocytes after 10-12 days (Hawking et al., 1971; Ngotho et al., 2019). While gametocytes represent the only cell type that is infectious to mosquitoes, they are non-replicative. Investments into gametocytogenesis thus come at the expense of reduced vegetative growth and $P$. falciparum employs sophisticated mechanisms to regulate this trade-off between within-host replication and between-host transmission (Carter et al., 2013).

Sexual commitment requires expression of AP2-G - a member of the ApiAP2 family of DNA-binding factors (Kafsack et al., 2014; Sinha et al., 2014). This master regulator of sexual commitment primes asexually replicating parasites to produce sexually committed ring stage progeny that exit the cell cycle and undergo gametocyte development (Josling et al., 2018). In addition to this mechanism referred to as 'next cycle conversion', the immediate induction of gametocytogenesis in ring stages has also been observed, albeit this 'same cycle conversion 'is induced at a low rate and has so far only been described in vitro (Bancells et al., 2019). In the predominant next cycle conversion route, the decision of whether to stay within the asexual pathway or commit to the production of gametocytes is made in early schizonts at $36+/-4$ hours post-erythrocyte invasion (hpi) (Brancucci et al., 2017). While AP2-G expression is initiated almost simultaneously, gametocyte differentiation will only start after completion of the current intra-erythrocytic developmental cycle (IDC) and the invasion of sexually committed merozoites into new RBCs (Ngotho et al., 2019). On the molecular level, the process of sexual commitment is under epigenetic control. It involves the activity of a number of well-characterized factors, including heterochromatin protein 1 (HP1), histone deacetylase 2 (HDA2) and gametocyte development protein 1 (GDV1) (Josling et al., 2018). These factors act in concert to control transcriptional activity at the ap2-g locus. The ap2-g locus is generally kept in a silenced state marked by the presence of histone 3 tri-methylated at lysine 9 (H3K9me3) and the histone code eraser and reader proteins HDA2 and HP1, respectively (Flueck et al., 2009; Lopez-Rubio et al., 2009; Salcedo-Amaya et al., 2009; Brancucci et al., 2014; Coleman et al., 2014). In contrast, GDV1 counteracts the silencing of $a p 2-g$ by evicting HP1 from the ap2-g locus, thus triggering AP2-G expression and sexual commitment in schizonts (Eksi et al., 2012; Filarsky et al., 2018; Usui et al., 2019). Through an auto-regulatory positive feedback loop, AP2$\mathrm{G}$ expression increases further and peaks in the sexually committed ring stage parasites where it binds to and regulates the expression of genes linked to gametocytogenesis (Bancells et al., 2019; Josling et al., 2020; Llorà-Batlle et al., 2020). Together, these studies revealed that sophisticated epigenetic mechanisms are in place to balance asexual reproduction versus investments into transmission stage formation (Carter et al. 2013; Josling et al., 2018).

$P$. falciparum parasites invest surprisingly little into transmission. In fact, sexual commitment rates (SCRs) in parasite populations typically remain at a low single-digit percentage in vitro and in vivo (Taylor and Read, 1997; Buchholz et al., 2011; Carter et al., 2013; Brancucci et al., 2015; Usui et al., 2019). This reproductive restraint can be lifted under certain circumstances, resulting in greatly enhanced sexual commitment of parasites and conversion rates above 30\% under specific in vitro conditions (Neveu et al., 2020). These include, but are not restricted to, high parasite densities (Bruce et al., 1990; Delves et al., 2016), exposure to P. falciparumconditioned (nutrient-depleted) medium (Williams, 1999; Dyer and Day, 2003; Fivelman et al., 2007; Brancucci et al., 2015), endoplasmic reticulum stress (Chaubey et al., 2014) or the uptake of extracellular vesicles derived from infected RBCs (iRBCs) (Mantel et al., 2013; Regev-Rudzki et al., 2013). These observations contributed to the appreciation that intraerythrocytic parasites are able to interact with and respond to their environment. Indeed, recent studies revealed that blood stage parasites modulate specific transcriptional programs in response to nutrient availability and other environmental cues (Kumar et al., 2021). Importantly, P. falciparum was found to metabolize the host-derived serum lipid lysophosphatidylcholine (lysoPC) in the Kennedy pathway and to induce sexual commitment under conditions that limit activity of this metabolic route (Brancucci et al., 2017). Expression of GDV1, the earliest known marker of sexual commitment, is induced at low lysoPC concentrations, implying a direct link between the epigenetic mechanisms that control gametocyte production and parasite nutrient-sensing (Neveu et al., 2020).

In addition, several lines of evidence suggest that some antimalarial drugs may interfere with the process of sexual commitment (Buckling et al., 1997; Puta and Manyando, 1997; Buckling et al., 1999a; Peatey et al., 2009; Baker, 2010; Portugaliza et al., 2020). Increased gametocyte production was observed in vitro upon treatment with artemisinin, mefloquine, chloroquine, primaquine, atovaquone and piperaquine (Peatey et al., 2009). 
Furthermore, treatment with sub-curative doses of the widely used drugs chloroquine (Buckling et al., 1997) and sulfadoxine/ pyrimethamine ("Fansidar") (Puta and Manyando, 1997) have been associated with increased gametocyte production in vivo. Using a transgenic reporter cell line for the quantification of sexual conversion rates (Portugaliza et al., 2019), the Cortés laboratory recently confirmed a gametocytogenesis-inducing effect for the frontline antimalarial dihydroartemisinin (DHA) (Portugaliza et al., 2020). In this study, short pulses of subcurative DHA concentrations applied to trophozoites induced sexual commitment in vitro, but this effect was not observed when ring or schizont stage parasites were exposed to the drug. Together, these studies raise legitimate concerns about whether therapeutic interventions may promote gametocytogenesis and hence malaria transmission. However, the mechanisms underlying such potential drug-induced increases in gametocyte production are hitherto unknown. On the one hand, antimalarials may promote sexual commitment by inducing general cellular stress responses. On the other hand, it is conceivable that gametocytogenesis may be induced by drug-specific modulation of the molecular process underlying sexual commitment - i.e. independent of the toxic effect of the drug. In this latter scenario, drug-resistant parasites would be expected to shift their investment towards gametocyte formation under drug pressure, which may eventually promote transmission and spread of drug resistance (White, 2008). Indeed, strains carrying specific drug resistance mutations have been associated with increased mosquito transmission following antimalarial treatment (Bell et al., 2012). Furthermore, chloroquine-resistant parasites showed increased gametocytaemia and mosquito infectivity following drug treatment compared to chloroquine-sensitive strains (Hogh et al., 1998). Other efforts, however, failed to confirm the specific induction of gametocyte production in drug-resistant parasites after exposure to sub-curative chloroquine and pyrimethamine concentrations in vitro (Reece et al., 2010). Hence, while drug-resistant parasite strains may have an increased transmission potential, it remains unclear whether this would be linked to higher parasite survival rates under drug pressure or truly increased rates of commitment to gametocyte formation.

The SCR reflects the proportion of schizonts within a given IDC that commit to gametocytogenesis and produce sexual ring stage progeny. Accurate calculation of SCRs hence relies on the simultaneous quantification of either asexually and sexually committed schizonts in the commitment cycle or of asexual parasites and early stage gametocytes in the immediate progeny, i.e. within the first 48 hours after invasion. However, since gametocytes are morphologically indistinguishable from asexual parasites until day three of sexual differentiation (stage II), the reliable determination of SCRs has traditionally been very laborious and technically challenging. Several laboratories have therefore developed flow cytometry-based assays that allow for an accurate measurement of gametocytaemia in parasites expressing fluorophores or fluorophore-tagged gametocyte markers under the control of ectopic gametocyte-specific promoters (Dixon et al., 2009; Peatey et al., 2009; Buchholz et al., 2011; Brancucci et al., 2015; Portugaliza et al., 2019). These assays enable distinguishing gametocytes from asexual stage parasites before they become morphologically distinct and therefore allow minimizing effects that may confound a precise determination of SCRs. Potential confounding factors include (i) the erroneous counting of gametocytes originating from multiple previous IDCs, (ii) the effect of multiplying asexual parasites and, in case of probing drugs or drug-like molecules, (iii) the potential lack of activity of compounds on early gametocyte survival. Because of the reduced sensitivity of gametocytes to most antimalarials (Peatey et al., 2012; Plouffe et al., 2016), it is important to identify and exclude confounding effects emerging from differential survival between asexual and sexual stage parasites. Hence, compared to standard light microscopybased setups, flow cytometry-based assays greatly improved the accuracy and likewise the throughput of measuring SCRs in parasite populations (Dixon et al., 2009; Peatey et al., 2009; Buchholz et al., 2011; Brancucci et al., 2015; Portugaliza et al., 2019).

Here, we developed a novel high content imaging (HCI) assay for the precise quantification of sexual commitment rates in $P$. falciparum parasites. This assay identifies sexually committed ring stage parasites based on the expression of endogenous mScarlet-tagged AP2-G, the earliest and most specific marker for sexually committed ring stages (Kafsack et al., 2014; Sinha et al., 2014; Bancells et al., 2019; Llorà-Batlle et al., 2020). Given the potential impact of antimalarials on malaria transmission, we used this assay to test a comprehensive panel of drugs for their possible effects on sexual commitment. In addition, we also included a diverse collection of other therapeutics that are commonly prescribed in malaria-endemic regions, including antihelminthics and analgesics, to account for a possible role of general stress-inducing factors on the sexual commitment process. Our results provide a systematic evaluation of the links between drug treatment and sexual commitment and suggest that antimalarial drug treatment does not promote transmission stage formation in P. falciparum.

\section{MATERIALS AND METHODS}

\section{Parasite Culture}

Intra-erythrocytic $P$. falciparum stages were cultured and synchronized as described (Jensen and Trager, 1978; Lambros and Vanderberg, 1979). Generally, parasites were grown in $\mathrm{AB}+$ or $\mathrm{B}+$ human RBCs (Blood Donation Center, Zürich, Switzerland) at a hematocrit of $5 \%$ in parasite culture medium consisting of $10.44 \mathrm{~g} / \mathrm{L}$ RPMI-1640, $25 \mathrm{mM}$ HEPES, $100 \mu \mathrm{M}$ hypoxanthine, $24 \mathrm{mM}$ sodium bicarbonate, 0.5\% AlbuMAX II (Gibco \#11021-037) and 0.1 g/L neomycin. The medium was further complemented with $2 \mathrm{mM}$ choline chloride (Sigma \#C7527) to maintain low background sexual commitment rates as observed in the presence of human serum (Brancucci et al., 2017). Cultures were gassed with $3 \% \mathrm{O}_{2}, 4 \% \mathrm{CO}_{2}$ and $93 \% \mathrm{~N}_{2}$ and incubated in an airtight incubation chamber at $37^{\circ} \mathrm{C}$. 


\section{Cloning of Transfection Constructs}

CRISPR/Cas9-based genome engineering of the NF54/ap2gmScarlet, TM90C2B/ap2g-mScarlet and NF54/ap2g-re9h parasites was performed using a two-plasmid approach as previously described (Brancucci et al., 2017; Filarsky et al., 2018). This system is based on co-transfection of a suicide and a donor plasmid. The suicide plasmid contains the expression cassettes for the Cas9 enzyme, the single guide RNA (sgRNA) and the human dihydrofolate reductase (hDHFR) resistance marker ( $\mathrm{pH}-\mathrm{gC}$ ). A pD-derived donor plasmid was used for homology-directed repair of the Cas9-induced DNA double strand break (Filarsky et al., 2018).

The pH-gC_ap2g-3' suicide plasmid targeting the 3' end of pfap2- $g$ has been described previously (Brancucci et al., 2017). The donor plasmid pD_ap2g-mScarlet was generated by assembling (i) the BamHI and SfoI-digested pD_ap2g-gfp plasmid (Brancucci et al., 2017) with a PCR product containing (ii) the mScarlet sequence preceded by nucleotides encoding a GSAG linker using the primers $\mathrm{mScarlet}-\mathrm{F}$ and $\mathrm{mScarlet}-\mathrm{R}$ amplified from a $P$. falciparum codon-optimized synthetic mScarlet sequence (Boltryk et al., 2021), and (iii) the 3 ' homology region amplified from the pD_ap2g-gfp plasmid (Brancucci et al., 2017) using the primers ap2-g-3'-F and ap2-g-3'-SfoI-R in a Gibson reaction (Gibson et al., 2009). The donor plasmid pD_ap2g-re9h was generated by assembling two PCR products using the primers iso_re9h-F and iso_re9h- $\mathrm{R}$ amplified from $\mathrm{pD}$ _ap2g-mScarlet, and (Gutman et al., 2009) re9h-F and re9h-R to amplify the re9h fragment (Branchini et al., 2010) from pTRIX2-re9h (Lewis et al., 2014). The sequence of the self-cleaving peptide T2A was included in the primers iso_re9h-R and re9h-F (Liu et al., 2017). Primer sequences used for cloning are listed in Table S1.

\section{Transfection and Selection of Gene Edited Parasites}

P. falciparum transfection using the CRISPR/Cas9 suicide and donor plasmid approach was performed as described previously (Filarsky et al., 2018). Briefly, $50 \mu \mathrm{g}$ of each of the suicide plasmid (pH-gC_ap2g-3') and the respective donor plasmid (pD_ap2g$\mathrm{mScarlet}$ or $\mathrm{pD} \_$ap2g-re9h) were co-transfected. Transgenic parasites were selected with $4 \mathrm{nM}$ WR $9921024 \mathrm{~h}$ after transfection for 6 days. Transgenic populations were usually obtained 2-3 weeks after transfection and correct editing of the ap2-g locus was then confirmed by PCR on gDNA. Primer sequences used for these PCRs are listed in Table S1.

\section{Drug Assays}

The screening for sexual commitment-inducing compounds was performed in a 96-well plate format and compounds were tested in twelve concentrations using two-step or three-step serial dilutions. One well per condition was measured for each independent biological replicate. Stock solutions of $10 \mathrm{mM}$ drug were prepared in DMSO, except for chloroquine, acetaminophen, Aspirin, diclofenac, ibuprofen and piparaquine (prepared in -SerM). Working solutions were prepared in -SerM medium immediately before the experiment and $100 \mu \mathrm{L}$ each was dispensed into the wells of a cell culture plate (Corning Incorporated, 96-well cell culture plate, flat bottom, REF 3596). Synchronous asexual parasites at 20$26 \mathrm{hpi}$ and a parasitaemia of $0.5-1 \%$ were washed and resuspended in -SerM medium complemented with $4 \mathrm{mM}$ choline chloride at $2.5 \%$ hematocrit. $100 \mu \mathrm{L}$ parasite suspension was then added to each well and gently mixed with the compounds. As a positive control for sexual commitment-inducing conditions, parasites resuspended in -SerM medium lacking choline chloride were used. Plates were gassed and incubated in an airtight incubation chamber at $37^{\circ} \mathrm{C}$ for 48 hours.

\section{Quantification of Parasite Survival}

At 20-26 h after reinvasion into new RBCs (i.e. 48 hours after the start of the assay), the parasitaemia was determined using flow cytometry. To this end, $40 \mu \mathrm{l}$ parasite suspension was transferred from each well to a new 96-well plate (Corning Incorporated, 96well cell culture plate, round bottom, REF 3788) and the samples were stained for 20 min with $40 \mu \mathrm{l}$ 2X SYBR Green DNA stain (Invitrogen S7563) and then washed twice in $200 \mu \mathrm{l}$ PBS. Plates were spun at $280 \mathrm{~g}$ for $2 \mathrm{~min}$ in between each step. To determine the parasitaemia, 200'000 events per sample were measured using the MACS Quant Analyzer 10. Data was analyzed using the FlowJo_v10.6.1 software. The gating strategy removed small debris and doublets (two cells per measurement), and iRBCs were distinguished from uninfected RBCs based on the SYBR Green intensity (Figure S6). Mean survival rates were calculated relative to -SerM/choline controls

$$
\frac{\text { parasitemia (test condition) }}{\text { parasitemia }(- \text { SerM/choline })} * 100
$$

from three independent biological replicates. Curve fitting was performed using non-linear, four parameter regression model with variable slope (Graph Pad Prism, version 8.2.1).

\section{Quantification of Sexual Commitment Rates by High Content Imaging}

The SCR is defined as the proportion of sexually committed parasites in the total population. High content imaging (HCI) microscopy and automated image analysis were used to detect the number of all parasites based on DNA staining alone, whereas the sexually committed parasites were recognized via both DNA staining and AP2-G-mScarlet fluorescence. At 20-26 hpi after reinvasion into new RBCs (i.e. 48 hours after the start of the assay), cultures were stained with Hoechst $(2.5 \mu \mathrm{g} / \mathrm{mL})$ for $20 \mathrm{~min}$ and washed twice in $200 \mu \mathrm{L}$ PBS. Plates were spun at $280 \mathrm{~g}$ for 2 $\mathrm{min}$ in between each step. The cultures were then diluted in PBS to a hematocrit of $0.075 \%$ and $200 \mu \mathrm{L}$ were transferred to a clearbottom 96-well HCI plate (Greiner CELLCOAT microplate 655948, Poly-D-Lysine, flat $\mu$ Clear bottom). Cells were allowed to settle for $15 \mathrm{~min}$ before image acquisition with an ImageXpress Micro widefield high content screening system (Molecular Devices) in combination with the MetaXpress software (version 6.5.4.532, Molecular Devices) and a Sola SE solid state white light engine (Lumencor). Filtersets for Hoechst (Ex: 377/50 nm, Em: 447/60 $\mathrm{nm}$ ) and $\mathrm{mScarlet}(\mathrm{Ex}: 543 / 22 \mathrm{~nm}$, Em: 593/40 nm) were used with exposure times of $80 \mathrm{~ms}$ and $600 \mathrm{~ms}$, respectively. 36 sites per well were imaged using a Plan-Apochromat 40x objective (Molecular 
Devices, cat\# 1-6300-0297). Automated image analysis was performed using the MetaXpress software (version 6.5.4.532, Molecular Devices). Hoechst-positive as well as mScarlet-positive parasites were identified using a modular image analysis workflow built within the ImageXpress software (Molecular Devices) described in Table S2, allowing for the calculation of sexual commitment rates (i.e. the proportion of Hoechst $/ \mathrm{mScarlet}$ double-positive cells among all Hoechst-positive cells). SCRs were calculated as follows

$$
\frac{m \text { Scarlet }- \text { positive cells }(\text { test condition })}{\text { Hoechst - positive cells }(\text { test condition })} * 100
$$

Mean relative SCRs were calculated relative to -SerM controls

$$
\frac{\operatorname{SCR}(\text { test condition })}{\operatorname{SCR}(-\operatorname{Ser} M)} * 100
$$

from three independent biological replicates.

\section{Quantification of RE9H Luminescence Intensity}

At 20-26 h after reinvasion into new RBCs (i.e. 48 hours after the start of the assay), $180 \mu \mathrm{L}$ of NF54/ap2g-re9h parasite culture was transferred from each well to a black 96-well plate (Greiner CELLSTAR microplate 655086, F-bottom, black) and incubated with $20 \mu \mathrm{L} \mathrm{D}$-Luciferin $(3.75 \mathrm{mg} / \mathrm{mL}$ in PBS) (Perkin Elmer, catalog\# 122799) for 5-10 $\mathrm{min}$ at room temperature. Luminescence intensities were then measured using an intra-vital imaging system (Perkin Elmer, Lumina II) by exposing the plates for $3 \mathrm{~min}$. Luminescence counts per well were determined by fitting a grid over the plate using the software Living Image (version 4.7.2).

\section{Quantification of Gametocyte Production Rates by Light Microscopy}

At 20-26 hpi, parasites were exposed to either -SerM or -SerM/ choline conditions, gassed and incubated in an airtight incubation chamber at $37^{\circ} \mathrm{C}$ for 48 hours to complete the IDC and produce ring stage progeny. At this time point, parasitaemia was determined using Giemsa-stained blood smears (day 1). The culture medium was replaced with culture medium containing $2 \mathrm{mM}$ choline and $50 \mathrm{mM} \mathrm{N}$-acetyl-D-glucosamine to eliminate asexual parasites and the cultures were incubated for 72 hours with daily medium changes (Fivelman et al., 2007). Gametocytaemia was determined by Giemsa-stained blood smears on day 4 (stage II gametocytes). Sexual commitment rates were calculated by dividing the gametocytaemia determined on day 4 by the total parasitaemia determined on day 1 using results obtained from three independent biological replicates.

\section{RESULTS}

\section{An Assay to Quantify Sexual Commitment Rates in $P$. falciparum}

De-repression of the ap2-g locus marks the earliest known transcriptional event of sexual commitment. Here, we used a
CRISPR/Cas9 gene editing strategy to fuse the ap2-g gene in frame to a sequence coding for the red fluorescent protein mScarlet (Figure 1A and Supplementary Figures 1A, B). Using these NF54/ap2g-mScarlet parasites, we established an HCI-based assay to quantify the SCRs in live parasite populations via monitoring expression of the fluorescently tagged AP2-G-mScarlet reporter protein (Supplementary Figure 1C). In brief, the sexual commitment assay is initiated by seeding highly synchronous NF54/ap2g-mScarlet parasites in 96-well flat-bottom cell culture plates at the late ring/early trophozoite stage (20-26 hpi) at $1.25 \%$ haematocrit and $0.5-1 \%$ parasitaemia and exposing them to test conditions. After a 48hour incubation period, i.e. during early intra-erythrocytic development of the subsequent generation, parasites are stained using the DNA dye Hoechst and transferred to a 96well imaging plate at a haematocrit of $0.075 \%$. The proportion of sexually committed ring stages/early stage I gametocytes (AP2G-mScarlet-positive) among all iRBCs (Hoechst-positive) is quantified by fluorescence HCI microscopy. Imaging of 36 sites per well allows capturing information for 5000-10`000 iRBCs. (Figure 1B). At the same time point, parasitaemia is determined for each well of the cell culture plate in parallel by flow cytometry of SYBR Green-stained iRBCs to assess potential effects of the test conditions on parasite survival (multiplication).

To establish and validate this HCI assay, we used parasites cultured in serum-free medium (-SerM) either in presence or absence of choline, the commitment-inhibiting metabolite of lysoPC (Brancucci et al., 2017). As expected, these +/- choline control conditions (-SerM/choline; -SerM) resulted in consistently low and high SCRs, respectively (Figure 1C), and revealed high assay robustness throughout the experiments presented here ( $Z^{\prime}$-factor of 0.79 (SD: 0.09), Figure 1D).

We used serum-free medium for two main reasons: First, the -SerM and -SerM/choline conditions allow for a controlled experimental setup across biological replicate experiments and, compared to serum-complemented medium, are independent of confounding factors such as varying levels of lysoPC and choline in serum deriving from different donors. Second, the balance between cytotoxic effects of lysoPC and activity on $P$. falciparum sexual commitment depends on a physical interplay between the lipid and serum albumin, which is difficult to reproduce in vitro (Kim et al., 2007; Brancucci et al., 2017). Therefore, and notwithstanding the fact that -SerM and -SerM/choline conditions cannot fully simulate in vivo conditions either, we decided to use serum-free medium for all experiments that quantify parasite sexual commitment in this study.

Importantly, SCRs determined by this HCI assay are highly consistent with the corresponding gametocyte formation rates observed by light microscopy of Giemsa-stained blood smears prepared three days after the HCI-based readout (Figure 1E). In absolute numbers, the NF54/ap2g-mScarlet line showed a mean SCR of $31.7 \%$ (95\% CI: 27.5-36.9\%) under inducing conditions (-SerM) and a baseline SCR of 5.2\% (95\% CI: 2.5-7.6\%) under commitment-repressing conditions (-SerM/choline). To further validate assay performance, we performed choline titration experiments that revealed half-maximal inhibition of sexual 
A NF54/ap2g-mScarlet
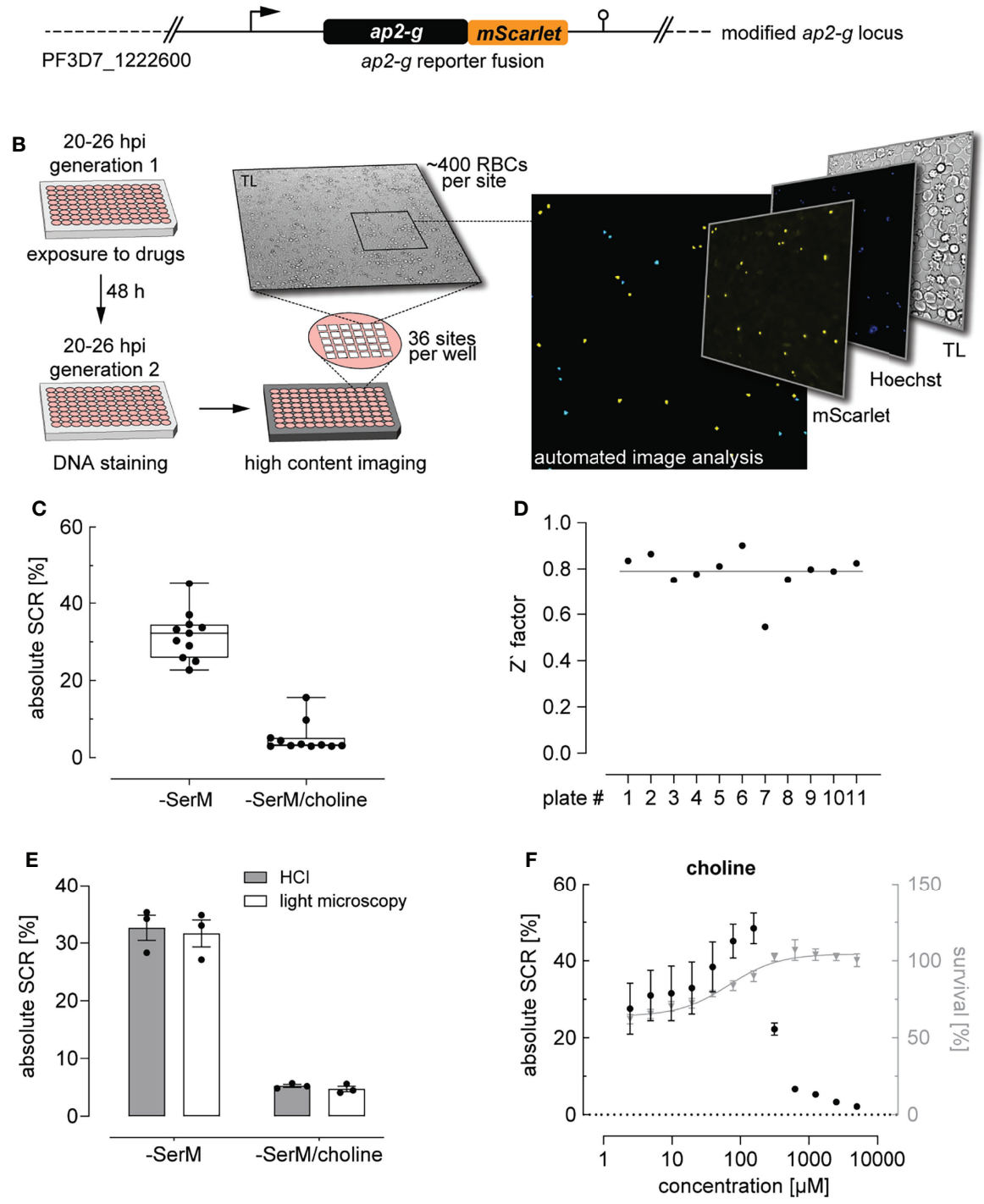

FIGURE 1 | High content imaging-based quantification of SCRs. (A) Schematic of the modified ap2-g locus in NF54/ap2g-mScarlet parasites. mScarlet, red fluorescent protein. (B) Experimental setup of the high content imaging-based sexual commitment assay. TL, transmitted light. (C) Rates and variation of sexual commitment in NF54/ap2g-mScarlet parasites under SCR-inducing (-SerM) and inhibiting (-SerM/choline) control conditions. Boxplots show interquartile ranges; whiskers mark minimal and maximal values. Black bullets represent the mean SCRs measured in individual wells (obtained from 6 wells per screening plate) for eleven independent biological replicate experiments. (D) Z factors determined for each screening plate. $n=11$; the line indicates the mean SCR across all plates. (E) SCRs and gametocyte formation rates under SCR-inducing (-SerM) and inhibiting (-SerM/choline) conditions quantified by high content imaging (HCI) and by light microscopy of stage II gametocytes on Giemsa-stained blood smears. $n=3$, error bars represent the standard error of the mean. (F) Dose-response effect of choline on the SCR (black bullets) and parasite survival (grey triangles). Data points represent the mean of three independent biological replicate experiments. Error bars represent the standard error of the mean.

commitment at $275 \mu \mathrm{M}$ choline $(95 \% \mathrm{CI}: 256-293 \mu \mathrm{M})$ and impaired parasite multiplication at low choline concentrations, which is consistent with previously published data (Brancucci et al., 2017) (Figure 1F).

In summary, these data show that this HCI-based assay allows capturing SCRs under controlled conditions and at a throughput that facilitates the systematic and robust investigation of modulators of parasite sexual commitment.

\section{Many Antimalarials Induce Sexual Commitment at Growth-Limiting Concentrations}

We used the above assay to investigate a total of 28 pharmaceuticals for potential effects on sexual commitment in 12 -point dose-response assays. In addition to 14 antimalarials, we also screened 14 drugs commonly used in malaria endemic regions, such as analgesics and antihelminthics (Table 1). 
TABLE 1 | Compounds tested for activities on $P$. falciparum sexual commitment.

\begin{tabular}{|c|c|c|c|}
\hline Antimalarials & effect on SCR reported in: & Drug class & $\mathrm{C}_{\max }$ \\
\hline amodiaquine & & quinoline & 1.8 нM (Ntale et al., 2009) \\
\hline atebrin & & acridine derivative & 125 nM (Croft, 2010) \\
\hline atovaquone ${ }^{\star}$ & (Peatey et al., 2009) & naphtoquinone & 68 M (Baggish and Hill, 2002) \\
\hline chloroquine* & $\begin{array}{l}\text { (Buckling et al., 1997; Buckling et al., 1999a; } \\
\text { Buckling et al., 1999b; Peatey et al., 2009) }\end{array}$ & 4-aminoquinoline & 500 nM (Mockenhaupt et al., 2000) \\
\hline dihydroartemisinin & (Portugaliza et al., 2020) & sesquiterpene lactone & $7 \mu \mathrm{M}$ (Saunders et al., 2014) \\
\hline lumefantrine & & arylamine alcohol & 530 nM (Ezzet et al., 2000) \\
\hline mefloquine* & (Peatey et al., 2009) & arylamine alcohol & $6 \mu \mathrm{M}$ (Gutman et al., 2009) \\
\hline piperaquine & (Peatey et al., 2009) & aminoquinoline & 750 nM (Hoglund et al., 2017) \\
\hline primaquine & (Peatey et al., 2009) & 8-aminoquinoline & 920 nM (Mello et al., 2018) \\
\hline proguanil & & dihydrofolate reductase inhibitor & 870 nM (Wattanagoon et al., 1987) \\
\hline pyrimethamine* & (Puta and Manyando, 1997) & aminopyrimidine & $2 \mu \mathrm{M}$ (Trenque et al., 2004) \\
\hline pyronaridine & & benzonaphthyridine derivative & 550 nM (Jittamala et al., 2015) \\
\hline quinine $^{\star}$ & & arylamine alcohol & 92 M (Flanagan et al., 2006) \\
\hline sulfadoxine & (Puta and Manyando, 1997) & sulfonamide & $3.2 \mu \mathrm{M}$ (Trenque et al., 2004) \\
\hline \multicolumn{4}{|l|}{ Antihelminthics } \\
\hline albendazole ${ }^{\star}$ & & benzimidazole & 233 nM (Schulz et al., 2019) \\
\hline ivermectin & & avermectin & 14.4 nM (Bernigaud et al., 2016) \\
\hline mebendazole & & benzimidazole & $1.6 \mu \mathrm{M}$ (Bekhti, 1985) \\
\hline moxidectin & & pentacyclic lactone & 463 nM (Cotreau et al., 2003) \\
\hline praziquantel & & pyrazoniquinoline & 2.6 M (Olliaro et al., 2014) \\
\hline \multicolumn{4}{|l|}{ Antipyretics } \\
\hline acetylsalicylic acid & & COX inhibitor & 422 M (Cerletti et al., 1984) \\
\hline ibuprofen & & nonsteroidal anti-inflammatory drug & 40 M (Mehlisch et al., 2010) \\
\hline acetaminophen & & nonsteroidal anti-inflammatory drug & 33.1 M (Saljoughian, 2016) \\
\hline diclofenac & & nonsteroidal anti-inflammatory drug & 51 nM (Miyatake et al., 2009) \\
\hline \multicolumn{4}{|l|}{ Antibiotics } \\
\hline azithromycin & & macrolide & 534 nM (Foulds et al., 1990) \\
\hline doxycycline & & tetracycline & 3.9 нM (Newton et al., 2005) \\
\hline \multicolumn{4}{|l|}{ Antidiabetics } \\
\hline gliquidone & & sulfonylurea & $1.2 \mu \mathrm{M}$ (von Nicolai et al., 1997) \\
\hline metformin & & guanidine & $14.3 \mu \mathrm{M}$ (Hess et al., 2018) \\
\hline \multicolumn{4}{|l|}{ Steroids } \\
\hline dexamethasone & & glucocorticoid & $6 \mu \mathrm{M}$ (Yang et al., 2008) \\
\hline
\end{tabular}

Studies that observed an elevating effect of antimalarials on gametocyte production are indicated. Drug classes and the maximum serum concentrations $\left(C_{\text {max }}\right)$ are shown. Asterisks mark compounds tested in three independent biological replicates.

Compounds showing SCR-inducing effects during primary screening were validated using three independent biological replicate experiments. Dimethyl sulfoxide (DMSO) was used as the vehicle for most compounds and did not show activity on parasite sexual commitment and survival at relevant concentrations $(<1 \%)$ (Supplementary Figure 2A).

While most antimalarials showed a trend towards elevating parasite SCR, this effect was restricted to a narrow concentration window and generally remained linked to drug levels that inhibited asexual parasite replication (Figure $\mathbf{2 A}$ and Supplementary Figure 2C). Chloroquine, pyrimethamine and mefloquine, for instance, had no prominent effect at low concentrations but elevated the parasite SCR just below the IC50 (Figure 2A and Supplementary Figure 2B). At higher drug concentrations, SCRs fluctuated markedly and reached high values in some instances. However, as these elevated SCRs were observed in populations with complete or near-complete inhibition of parasite survival (Figure 2A), they did not result in an enhanced production of sexually committed parasites. In fact, when corrected for parasite survival, only specific sub-IC50 concentrations of the widely used mefloquine and pyrimethamine led to a significant increase in sexual ring stage formation on the absolute scale (Figure 2B). While the effect of pyrimethamine was minor, mefloquine, which is known to inhibit protein synthesis via direct binding to $80 \mathrm{~S}$ ribosomes (Wong et al., 2017), increased the formation of ring stage gametocytes by a factor of 2.4 (95\% CI: 1.2 to 4.7$)$ at a concentration of $3.9 \mathrm{nM}$, i.e. several magnitudes below therapeutic concentrations (Karbwang et al., 1987; Gutman et al., 2009). It is conceivable that decreased translation rates in mefloquine treated parasites may serve as a signal for poor nutrient availability, which may in turn act as a cue to invest more resources into increased gametocyte formation in order to maximize host-to-host 

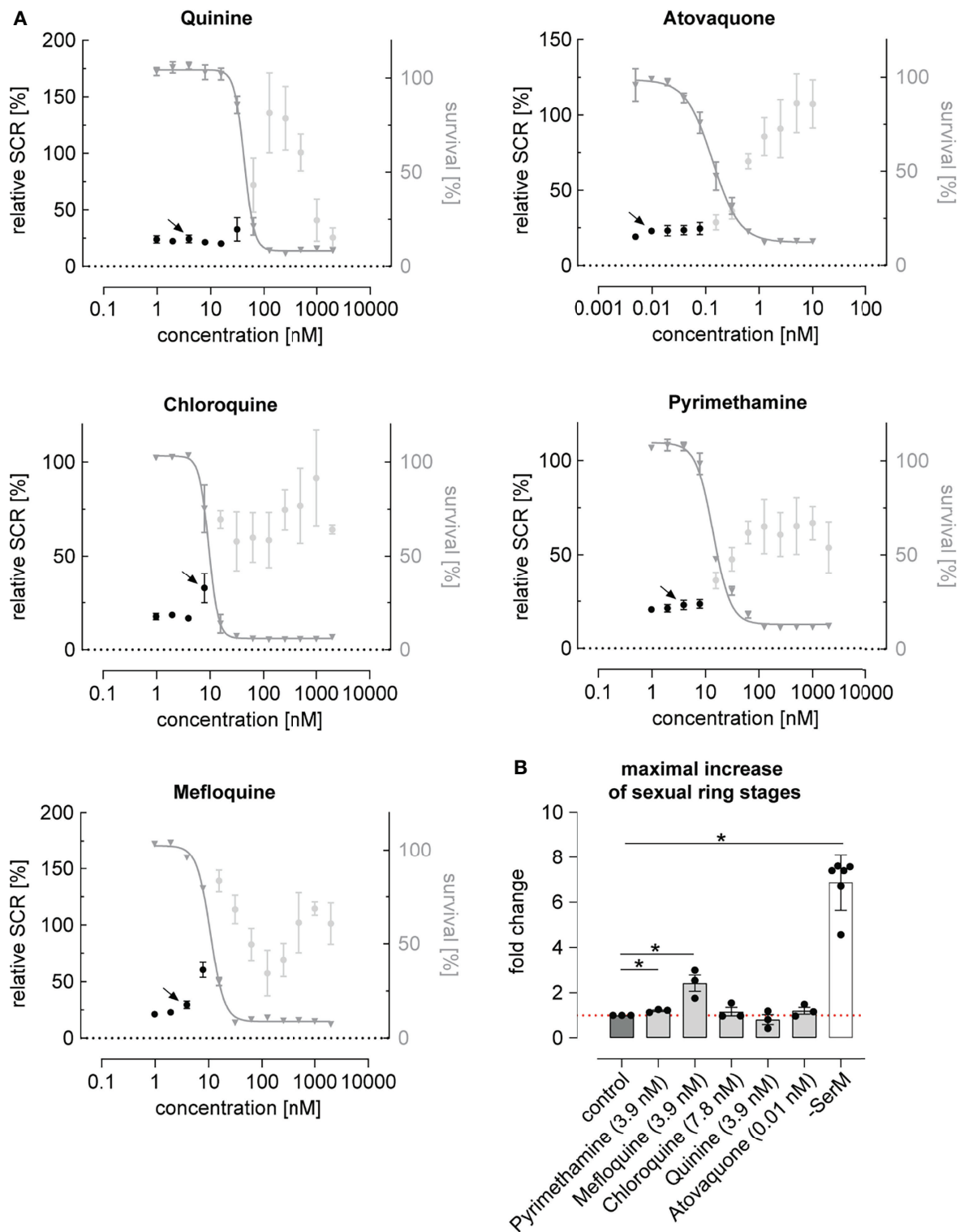

FIGURE 2 | Dose-response relationship between antimalarial compounds and parasite sexual commitment. (A) Antimalarials show a general trend towards increasing the proportion of sexually committed parasites at growth-inhibiting concentrations. Mean parasite survival rates and SCRs are indicated by grey triangles and black bullets, respectively. Grey bullets represent SCRs at compound concentrations above the IC50. Values are normalized to the corresponding control conditions (-SerM for SCR) and (-SerM/choline for survival). Data points represent the mean of three independent biological replicate experiments. Error bars represent the standard error of the mean. Arrows indicate data points used for the calculation of SCR fold-changes shown in (B). (B) Exposure to sub-therapeutic mefloquine concentrations result in an absolute increase of sexually committed ring stage progeny formed. Pyrimethamine shows a similar but markedly less pronounced effect. Bars indicate mean fold changes of sexual ring stage formation compared to untreated control conditions (-SerM/choline/DMSO), with black

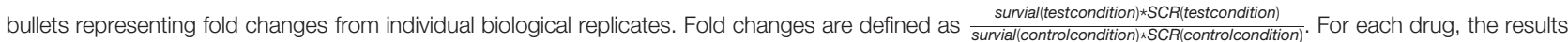
shown are derived from the concentration for which the maximal net increase in absolute sexual ring stage formation was observed. Drug concentrations are indicated. -SerM shows the effect of choline depletion on sexual ring stage formation. Asterisks mark significant differences ( $p$-value $<0.05$; paired two-tailed Student's t-test). $n=3$; error bars represent the standard error of the mean.

transmission under stress conditions (Carter et al., 2013). A possible link between translation inhibition and AP2-G expression, however, remains to be established.

Noteworthy, parasites treated with artemisinin or its derivatives dihydroartemisinin (DHA), artemether and artesunate, emitted autofluorescence at various wavelengths including the TRITC channel, making a fluorescence-based quantification of SCRs impossible. To circumvent this issue and evaluate the effect of DHA, for which a sexual commitmentinducing effect has recently been demonstrated (Portugaliza et al., 
2020), we generated the NF54/ap2g-re9h reporter line (Figure 3A and Supplementary Figure 3). These parasites express an AP2G-T2A-RE9H luciferase fusion protein from the endogenous ap2$g$ locus and hence allow using luminescence as a proxy for quantifying AP2-G expression (Figure 3B). In contrast to the fluorescence-based assay described above, the NF54/ap2g-re9h line enables determining absolute expression of the AP2-G-RE9H reporter protein in parasite populations per well rather than quantifying SCRs at a single cell level. The NF54/ap2g-re9h cell line showed robustness in reporting sexual commitment under control conditions (Z'-factor of 0.57 (SD: 0.13 ) as well as the ability to capture the dose-dependent effect of choline on SCRs (half-maximal inhibition of sexual commitment at $194 \mu \mathrm{M} ; 95 \%$ CI: 136-298 $\mu \mathrm{M})$ (Figure 3C).

After having validated the use of NF54/ap2g-re9h parasites for screening purposes, we made use of this line to quantify the effect of selected drugs on sexual commitment. These experiments confirmed the SCR-inducing effect of mefloquine at sub-therapeutic conditions (Figure 3D), and the lack thereof after treatment with atovaquone (Figure 3E), corroborating the results obtained with the NF54/ap2gmScarlet line (Figure 2A). Importantly, no increase in SCRs was observed following exposure to DHA (Figure 3E).

A NF54/ap2g-re9h

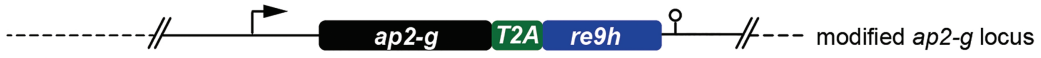

PF3D7_1222600 ap2-g reporter fusion

B

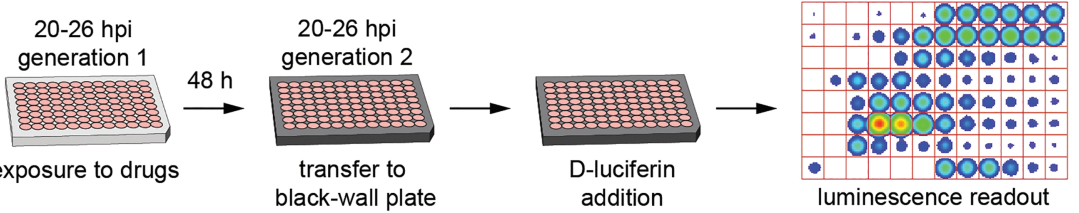

C

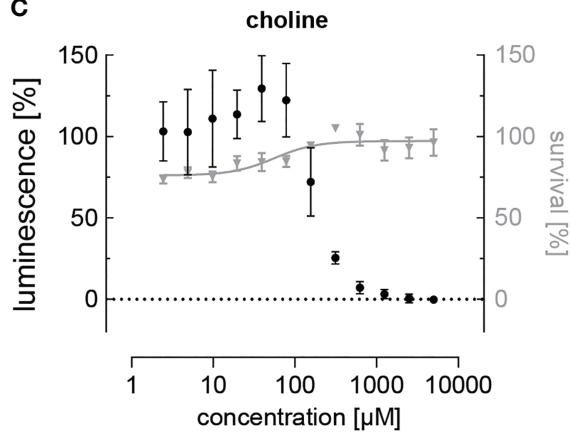

E

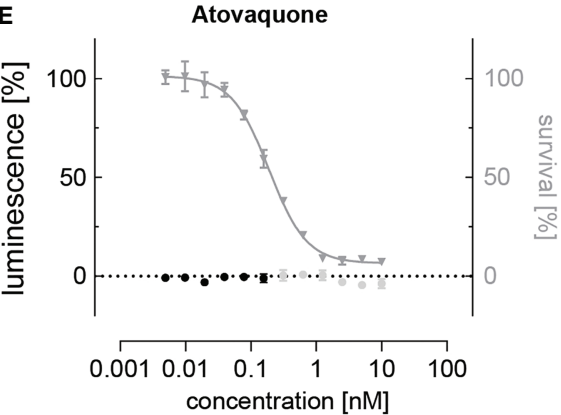

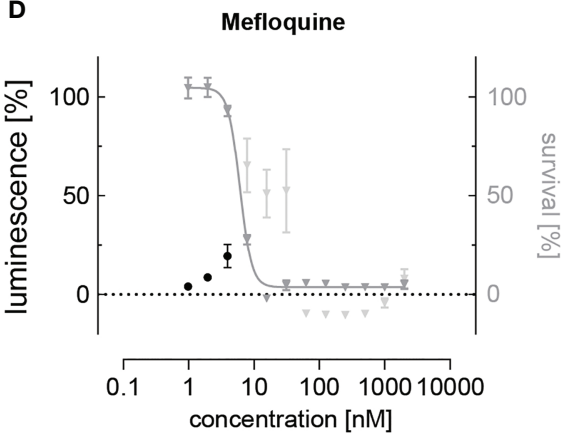

DHA

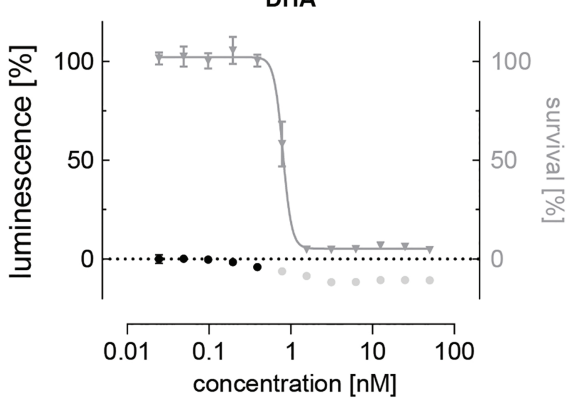

FIGURE 3 | Luciferase-based quantification of sexual commitment. (A) Schematic of the modified ap2-g locus in NF54/ap2g-re9h parasites. re9h, gene encoding red-shifted firefly luciferase RE9H. T2A, self-cleaving peptide. (B) Experimental setup of RE9H luciferase-based quantification of SCRs. (C) Dose-response effect of choline on the SCR (black bullets) and parasite survival (grey triangles). Data points represent the mean of three independent biological replicate experiments. Error bars represent the standard error of the mean. (D) Mefloquine induces sexual commitment within a narrow sub-therapeutic window. Mean parasite survival rates and relative RE9H reporter-mediated luminescence as a surrogate for SCRs are indicated by grey triangles and black bullets, respectively. Grey bullets represent SCRs at compound concentrations above the IC50. Values are normalized to the corresponding control conditions (-SerM for SCR) and (-SerM/choline for survival). $\mathrm{n}=3$; error bars represent the standard error of the mean. (E) Neither atovaquone nor DHA induce sexual commitment. Mean parasite survival rates and relative RE9H reportermediated luminescence as a surrogate for SCRs are indicated by grey triangles and black bullets, respectively. Grey bullets represent SCRs at compound concentrations above the IC50). Values are normalized to the corresponding control conditions (-SerM for SCR) and (-SerM/choline for survival). $n=3$; error bars represent the standard error of the mean. 


\section{Commonly Prescribed Drugs Have No Relevant Effect on Sexual Commitment}

Next, we investigated the effect of a collection of frequently used drugs, including antihelminthics, antibiotics as well as compounds used to treat pain, fever and inflammation (Table 1). Similar to what we observed for most antimalarials, antihelminthic drugs, including albendazole, ivermectin and moxidectin, showed trends towards increasing sexual commitment in the NF54/ap2g-mScarlet line (Figure 4A and Supplementary Figure 4). For most compounds, however, this effect was restricted to parasite growth-inhibiting drug concentrations that are substantially higher than the maximum serum levels observed in patients after standard treatment
(Cotreau et al., 2003; Newton et al., 2005; Bernigaud et al., 2016; Saljoughian, 2016; Hess et al., 2018; Schulz et al., 2019). Interestingly, moxidectin, a macrocyclic lactone known to be active against Plasmodium berghei mosquito stages (Azevedo et al., 2019), also prevented asexual parasite replication rather effectively (IC50 = $159 \mathrm{nM}$ ) (Figure 4B). While moxidectin induced sexual commitment at concentrations near the IC50, no net increase in ring stage gametocytes was observed. The tested antipyretics (aspirin, ibuprofen, diclofenac and acetaminophen), antibiotics (azithromycin and doxycycline), antidiabetics (metformin, gliquidone) as well as dexamethasone, a corticosteroid with anti-inflammatory and immunosuppressant properties, had no effects on parasite sexual commitment at
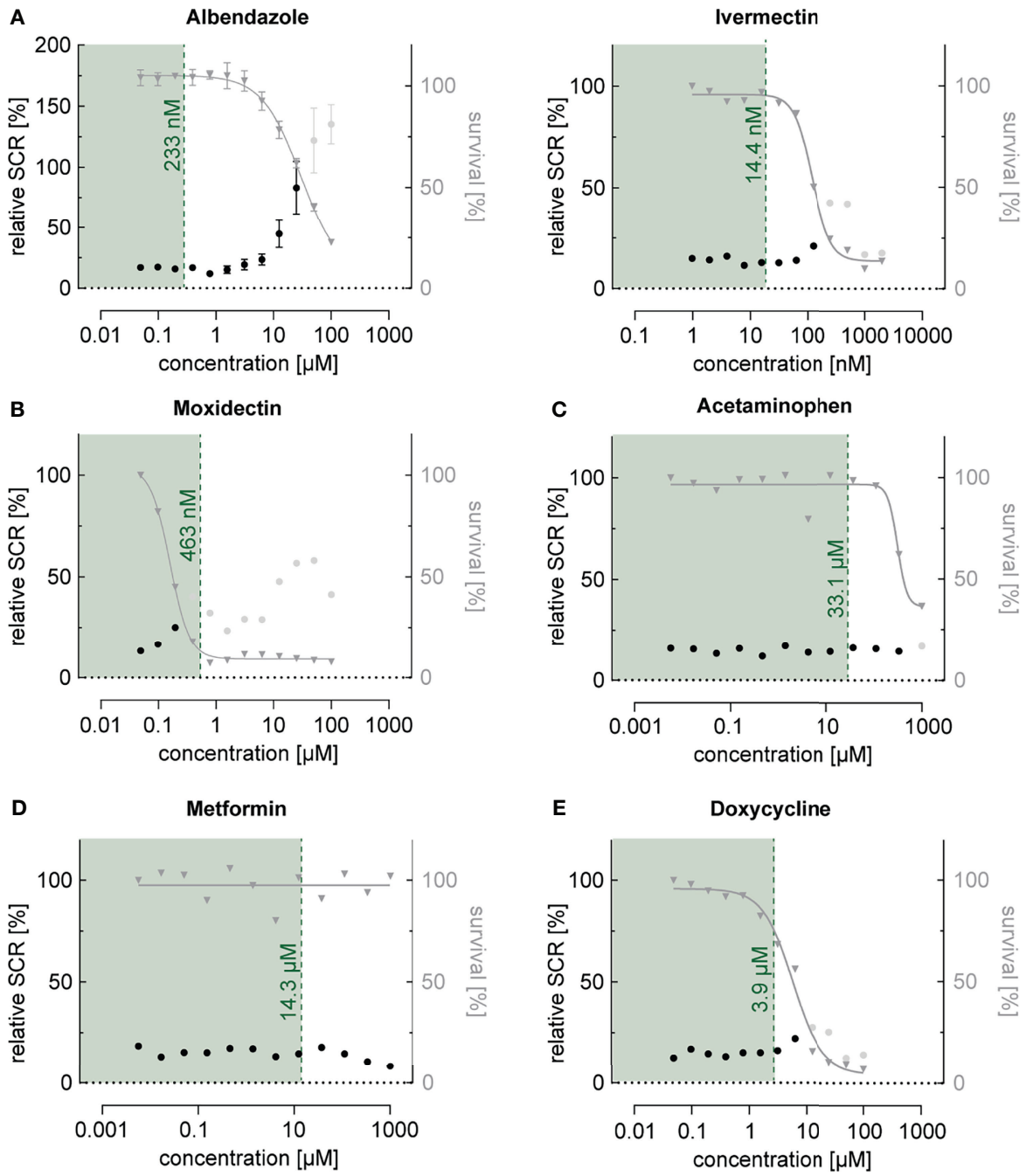

FIGURE 4 | Dose-response relationship between commonly used drugs and parasite sexual commitment. (A) The antihelminthics albendazole and ivermectin show a general trend towards induction of sexual commitment at super-physiological concentrations that also affect parasite growth (see also Figure S4). (B) The antihelminthic moxidectin affects parasite growth as well as SCRs at physiological concentrations. (C-E) The commonly used drugs acetaminophen (antipyretic) (C), metformin (antidiabetic) (D) and doxycycline (antibiotic) (E) have no effect on parasite growth or SCRs at physiologically relevant concentrations. Mean parasite survival rates and SCRs are indicated by grey triangles and black bullets, respectively. Grey bullets represent SCRs at compound concentrations above the IC50. The maximum physiological concentrations are indicated in green. Values are normalized to the corresponding control conditions (-SerM for SCR) and (-SerM/choline for survival). Albendazole: $n=3$; error bars represent the standard error of the mean. Ivermectin, Moxidectin, Acetaminophen, Metformin and Doxycycline: $n=1$. 
medically relevant concentrations (Cerletti et al., 1984; von Nicolai et al., 1997; Yang et al., 2008; Miyatake et al., 2009) (Figures 4C-E and Supplementary Figure 4).

\section{Cellular Stress Modulates Sexual Commitment Rather Than Target-Specific Activities}

Drug resistance has been associated with increased gametocyte carriage in malaria patients with uncomplicated P. falciparum infections (Price et al., 1999; Méndez et al., 2007; White, 2008; Bell et al., 2012). However, whether these observations are a consequence of a higher burden of asexually replicating parasites or higher rates of sexual commitment is unclear. To address this question, we tagged the ap2-g locus in the multidrug-resistant $P$. falciparum strain TM90C2B using the same CRISPR/Cas9 gene editing approach employed to generate the NF54/ap2g-mScarlet line (Figure 5A and Supplementary Figure 5). The TM90C2B strain has reduced susceptibility to chloroquine, cycloguanil, pyrimethamine and atovaquone (Chugh et al., 2015). As expected, transgenic parasites of the TM90C2B/ap2g-mScarlet cell line showed substantially higher tolerance to chloroquine and pyrimethamine compared to the non-resistant NF54/ap2g$\mathrm{mScarlet}$ control line (Figure 5B). For chloroquine, the IC50 increased approximately 8-fold from $9.4 \mathrm{nM}$ (95\% CI: 8.477 to $10.30 \mathrm{nM}$ ) to $71.2 \mathrm{nM}$ (95\% CI: 63.79 to $79.69 \mathrm{nM}$ ) and for pyrimethamine by a factor of $2 ` 500$ from $14.1 \mathrm{nM}$ (95\% CI: 12.79 to $15.42 \mathrm{nM}$ ) to $36.1 \mu \mathrm{M}$ (95\% CI: 22.9 to $175.9 \mu \mathrm{M}$ ). Similar to the observations made for NF54/ap2g-mScarlet parasites, chloroquine showed a dose-dependent trend towards increasing sexual commitment in the TM90C2B/ap2g-mScarlet line. Importantly, however, drug concentrations that led to increased SCRs in the drug-sensitive NF54/ap2g-mScarlet strain were ineffective in drugresistant TM90C2B/ap2g-mScarlet parasites. In these multidrugresistant parasites, increased SCRs were only observed at chloroquine concentrations that inhibited asexual replication beyond the IC50 (Figure 5B). Likewise, pyrimethamine, for which we observed a minor but statistically significant inducing effect on the formation of sexual ring stages (see Figure 2), elevated sexual commitment in TM90C2B/ap2g-mScarlet cells. Again, this activity was exclusively observed at drug concentrations that substantially inhibited parasite growth $(>10$ $\mu \mathrm{M})$ and in this case even exceeded the serum concentrations observed in patients following standard treatment (median of 400 nM (Trenque et al., 2004)).

\section{DISCUSSION}

Malaria transmission relies on the formation of gametocytes from a pool of asexually replicating parasites. The proportion of parasites that differentiate into these sexual sages, the so-called sexual commitment rate, is variable and - at least to a certain extent driven by cues in the microenvironment of intra-erythrocytic parasites (Williams, 1999; Dyer and Day, 2003; Fivelman et al., 2007; Peatey et al., 2009; Brancucci et al., 2015; Brancucci et al., 2017; Portugaliza et al., 2020). While a number of conditions, including exposure to antimalarial drugs, have been suggested or demonstrated to stimulate sexual commitment (Maswoswe et al., 1985; Buckling et al., 1997; Puta and Manyando, 1997; Drakeley et al., 2006; Babiker et al., 2008; Peatey et al., 2009; Baker, 2010;

A TM90C2B/ap2g-mScarlet

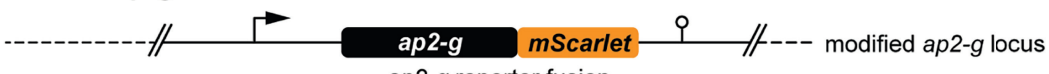

ap2-g reporter fusion

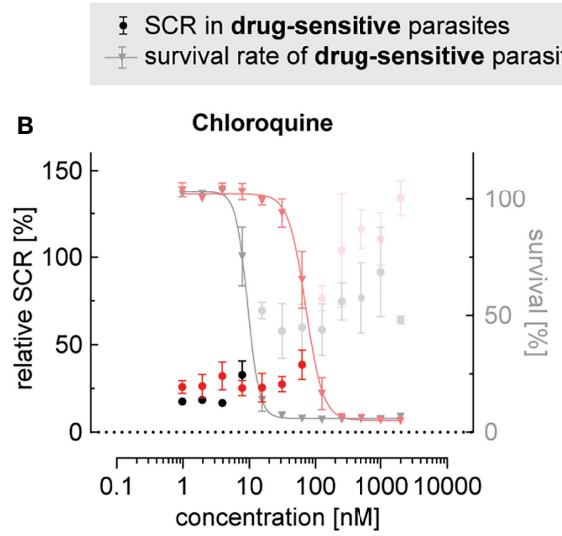

$\Phi$ SCR in drug-resistant parasites

survival rate of drug-resistant parasites

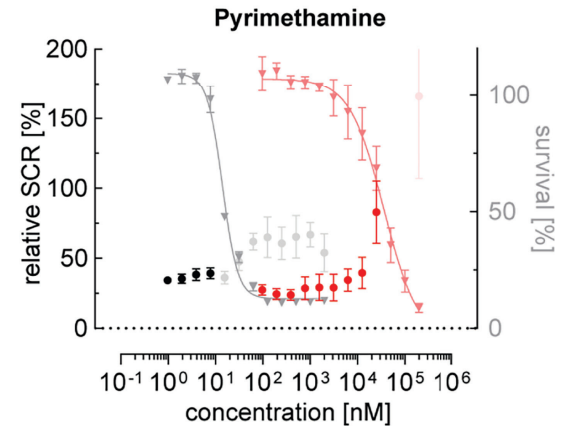

FIGURE 5 | Effect of antimalarials on SCR in drug-resistant parasites. (A) Schematic of the modified ap2-g locus in multidrug-resistant TM90C2B/ap2g-mScarlet parasites. (B) Trends towards increased sexual commitment are linked to growth-inhibiting concentrations of Chloroquine and Pyrimethamine in drug-sensitive as well as in drug-resistant parasite lines. For NF54/ap2g-mScarlet, mean parasite survival rates and SCRs are indicated by grey triangles and black bullets, respectively (values adopted from Figure 2A). For drug-resistant TM90C2B/ap2g-mScarlet parasites, mean parasite survival rates and SCRs are indicated by light red triangles and red bullets, respectively. Grey and light red bullets represent SCRs at compound concentrations above the IC50. Values are normalized to the corresponding control conditions (-SerM for SCR) and (-SerM/choline for survival). $\mathrm{n=3}$; error bars represent the standard error of the mean. 
Mantel et al., 2013; Regev-Rudzki et al., 2013; Chaubey et al., 2014; Brancucci et al., 2017; Portugaliza et al., 2020), the impact of therapeutic interventions on gametocyte production and malaria transmission is still a matter of debate. Differences in drugsusceptibility between $P$. falciparum asexual parasites and gametocytes, combined with the long period of sexual differentiation, render an evaluation of drug-induced effects on gametocytogenesis a challenging endeavor. Here, we established a high content imaging-based assay to systematically probe the impact of a comprehensive set of antimalarials and other drugs commonly prescribed in malaria-endemic regions on $P$. falciparum sexual commitment. Allowing for the simultaneous quantification of parasite survival and SCRs, this assay facilitated measuring net effects on gametocyte formation.

Consistent with previous observations, we found that $P$. falciparum parasites tend to elevate SCRs following in vitro exposure to a multitude of drugs, including, but not limited to antimalarials. While this effect was negligible for most tested compounds, some antimalarials showed a trend towards inducing the rate at which parasites committed to the sexual pathway. Chloroquine for example, elevated the SCR by a factor of 1.64 (95\% CI: 0.4-6.1) at a concentration of $7.8 \mathrm{nM}$ (see Supplementary Figure 2B) when compared to the untreated control population. This effect, however, was restricted to a narrow drug concentration window around the IC50 value and did not cause a net increase in sexual ring stages formed (see Figure 2B).

Contrary to this general trend, exposure to pyrimethamine and mefloquine did not only elevate the SCR but also caused a low to moderate net increase in absolute numbers of sexual ring stages, respectively. These gametocytogenesis-promoting activities were linked to specific concentrations (3.9 nM for pyrimethamine; 3.9 $\mathrm{nM}$ for pyrimethamine and mefloquine) near the IC50 for both drugs. While the 2.4-fold increase in sexual ring stages formed (95\% CI: 1.2-4.7) after exposure to $3.9 \mathrm{nM}$ mefloquine was the highest activity observed throughout this study, this value is substantially lower compared to the gametocytogenesis-promoting effect observed under LysoPC/choline-depleted -SerM control conditions (fold change of 7.3; 95\% CI: 6.1-8.7). Considering this relatively low drug-induced activity, as well as the narrow drug concentration window within which sexual commitment was elevated, it seems highly unlikely that drug treatment per se could have a relevant effect on promoting gametocyte production and malaria transmission in real life settings.

Nevertheless, our data reinforce the view that parasites can change rates of sexual commitment and probably also the absolute number of gametocytes formed in response to exposure to drugs at sub-curative levels. Fueled by previous reports about increased gametocytaemias and mosquito infectivity following treatment of parasites with drug resistance mutations (Hogh et al., 1998; Bell et al., 2012), our observations thus raise the question as to whether therapeutic drug concentrations could provoke a disproportionally high rate of gametocyte formation in drug-resistant parasites. Using the multidrug-resistant parasite strain TM90C2B, we could not observe such effects for chloroquine and pyrimethamine and their activities on SCRs remained tightly linked to growth-inhibiting drugs levels. For instance, while we found the TM90C2B parasites to elevate SCRs in response to pyrimethamine exposure, this activity occurred only at a drug concentration of $25 \mu \mathrm{M}$, i.e. at growth-inhibiting concentrations close to the IC50 and >2 500 -fold higher compared to the concentration that led to elevated SCRs in the pyrimethamine-sensitive NF54 strain.

The strict link between unfavorable growth conditions and elevated parasite SCRs strongly suggests that drug-induced sexual commitment is linked to general stress responses, rather than to compound-specific effects targeting the sexual commitment pathway. In fact, to date we are missing strong evidence for the ability of antimalarials or other drugs to interfere with the molecular process of variable gametocyte formation. Considering the involvement of epigenetic control mechanisms and phospholipid metabolism in the regulation of parasite sexual commitment (Brancucci et al., 2014; Coleman et al., 2014; Brancucci et al., 2017), it would however not be surprising to observe corresponding effects for drugs interfering with these processes specifically. For instance, histone deacetylase inhibitors, which have important applications in anti-cancer treatments (Eckschlager et al., 2017) and show promising activity against $P$. falciparum blood stage parasites (Andrews et al., 2012; Chua et al., 2017), may interfere with heterochromatin-mediated silencing of the ap2-g locus. Similarly, choline kinase inhibitors, for which a direct effect on parasite sexual commitment has previously been demonstrated (Brancucci et al., 2017), were proposed as new therapeutic tools against a variety of human diseases, including bacterial and parasitic infections (Lacal et al., 2021). It will thus be important to carefully evaluate potential effects of such molecules on sexual commitment and gametocyte formation before developing them into antimalarial agents.

Based exclusively on in vitro experiments, it is clear that the data presented here cannot fully reflect the complex situation found in patients infected with $P$. falciparum. For example, the different microenvironments that parasites encounter at sequestration sites, including the bone marrow and spleen, may have profound effects on drug kinetics and bioavailability. Furthermore, in recent efforts, Portugaliza and colleagues simulated the short in vivo half-life of artemisinin/DHA by exposing in vitro cultured parasites to short drug pulses and identified stage-specific effects on sexual commitment (Portugaliza et al., 2020). While ring stage populations exposed to 3-hour pulses of DHA responded with decreased SCRs, trophozoites showed elevated SCRs following drug pressure. By contrast, the experiments presented here did not reveal an effect of this artemisinin derivative on parasite SCR. These discrepancies are likely a result of the distinct experimental setups used - particularly the different periods of drug exposure used. Clearly, a comprehensive picture of physiologically relevant links between $P$. falciparum gametocyte production and drug pressure can only be gained by accounting for a variety of parameters including pharmacokinetics, pharmacodynamics as well as different host determinants and microenvironments. The limitations of our in vitro studies notwithstanding, the data presented here imply that none of the existing antimalarial drugs act specifically on the molecular pathways controlling sexual 
commitment and are hence unlikely to significantly enhance malaria transmission. This is in line with studies reporting that combination therapies, in particular ACTs, are associated with an effective reduction in gametocyte carriage (Okell et al., 2008; Ippolito et al., 2017; WHO., 2020) and indicates that the positive effect of antimalarial treatment clearly outweighs potential risks of increased transmission.

\section{DATA AVAILABILITY STATEMENT}

The original contributions presented in the study are included in the article/Supplementary Material. Further inquiries can be directed to the corresponding authors.

\section{AUTHOR CONTRIBUTIONS}

BT performed all experiments, analyzed, and interpreted the data. EH, AP, and BT generated the transgenic parasite lines. TB performed experiments performed with NF54/ap2g-re9h parasites. NB and TV conceived of the study, designed and supervised experiments, and provided resources. NB prepared illustrations and wrote the manuscript. TV edited the

\section{REFERENCES}

Andrews, K. T., Tran, T. N., and Fairlie, D. P. (2012). Towards Histone Deacetylase Inhibitors as New Antimalarial Drugs. Curr. Pharm. Des. 18 (24), 3467-3479. doi: 10.2174/138161212801327257

Azevedo, R., Mendes, A. M., and Prudêncio, M. (2019). Inhibition of Plasmodium Sporogonic Stages by Ivermectin and Other Avermectins. Parasit Vectors 12 (1), 549. doi: 10.1186/s13071-019-3805-0

Babiker, H. A., Schneider, P., and Reece, S. E. (2008). Gametocytes: Insights Gained During a Decade of Molecular Monitoring. Trends Parasitol. 24 (11), 525-530. doi: 10.1016/j.pt.2008.08.001

Baggish, A. L., and Hill, D. R. (2002). Antiparasitic Agent Atovaquone. Antimicrob. Agents Chemother. 46 (5), 1163-1173. doi: 10.1128/AAC.46.5. 1163-1173.2002

Baker, D. A. (2010). Malaria Gametocytogenesis. Mol. Biochem. Parasitol. 172 (2), 57-65. doi: 10.1016/j.molbiopara.2010.03.019

Bancells, C., Llorà-Batlle, O., Poran, A., Nötzel, C., Rovira-Graells, N., Elemento, O., et al. (2019). Revisiting the Initial Steps of Sexual Development in the Malaria Parasite Plasmodium Falciparum. Nat. Microbiol. 4 (1), 144-154. doi: 10.1038/s41564-018-0291-7

Bekhti, A. (1985). Serum Concentrations of Mebendazole in Patients With Hydatid Disease. Int. J. Clin. Pharmacol. Ther. Toxicol. 23 (12), 633-641.

Bell, A. S., Huijben, S., Paaijmans, K. P., Sim, D. G., Chan, B. H., Nelson, W. A., et al. (2012). Enhanced Transmission of Drug-Resistant Parasites to Mosquitoes Following Drug Treatment in Rodent Malaria. PloS One 7 (6), e37172. doi: 10.1371/journal.pone.0037172

Bernigaud, C., Fang, F., Fischer, K., Lespine, A., Aho, L. S., Dreau, D., et al. (2016). Preclinical Study of Single-Dose Moxidectin, a New Oral Treatment for Scabies: Efficacy, Safety, and Pharmacokinetics Compared to Two-Dose Ivermectin in a Porcine Model. PloS Negl. Trop. Dis. 10 (10), e0005030. doi: 10.1371/journal.pntd.0005030

Boltryk, S. D., Passecker, A., Alder, A., Carrington, E., van de Vegte-Bolmer, M., van Gemert, G. J., et al. (2021). CRISPR/Cas9-Engineered Inducible Gametocyte Producer Lines as a Valuable Tool for Plasmodium Falciparum Malaria Transmission Research. Nat. Commun. 12 (1), 4806. doi: 10.1038/ s41467-021-24954-4 manuscript. All authors contributed to the final editing of the manuscript.

\section{FUNDING}

This work was supported by the Swiss National Science Foundation (grants 310030_200683 and BSCGI0_157729) and the Fondation Pasteur Suisse.

\section{ACKNOWLEDGMENTS}

We thank Christian Scheurer for providing the multidrugresistant parasite line TM90C2B and Eilidh Carrington for supervising the engineering of the $\mathrm{pD} \_a p 2 \mathrm{~g}$-mScarlet plasmid.

\section{SUPPLEMENTARY MATERIAL}

The Supplementary Material for this article can be found online at: https://www.frontiersin.org/articles/10.3389/fcimb.2022.802341/ full\#supplementary-material

Branchini, B. R., Ablamsky, D. M., Davis, A. L., Southworth, T. L., Butler, B., Fan, F., et al. (2010). Red-Emitting Luciferases for Bioluminescence Reporter and Imaging Applications. Anal. Biochem. 396 (2), 290-297. doi: 10.1016/ j.ab.2009.09.009

Brancucci, N. M. B., Bertschi, N. L., Zhu, L., Niederwieser, I., Chin, W. H., Wampfler, R., et al. (2014). Heterochromatin Protein 1 Secures Survival and Transmission of Malaria Parasites. Cell Host Microbe 16 (2), 165-176. doi: 10.1016/j.chom.2014.07.004

Brancucci, N. M. B., Gerdt, J. P., Wang, C., De Niz, M., Philip, N., Adapa, S. R., et al. (2017). Lysophosphatidylcholine Regulates Sexual Stage Differentiation in the Human Malaria Parasite Plasmodium Falciparum. Cell 171 (7), 1532 44.e15. doi: 10.1016/j.cell.2017.10.020

Brancucci, N. M., Goldowitz, I., Buchholz, K., Werling, K., and Marti, M. (2015). An Assay to Probe Plasmodium Falciparum Growth, Transmission Stage Formation and Early Gametocyte Development. Nat. Protoc. 10 (8), 11311142. doi: 10.1038/nprot.2015.072

Bruce, M. C., Alano, P., Duthie, S., and Carter, R. (1990). Commitment of the Malaria Parasite Plasmodium Falciparum to Sexual and Asexual Development. Parasitology 100 Pt 2, 191-200. doi: 10.1017/S0031182000061199

Buchholz, K., Burke, T. A., Williamson, K. C., Wiegand, R. C., and Wirth, D. F. (2011). Marti MA High-Throughput Screen Targeting Malaria Transmission Stages Opens New Avenues for Drug Development. J. Infect. Dis. 203 (10), 1445-1453. doi: 10.1093/infdis/jir037

Buckling, A., Crooks, L., and Read, A. (1999a). Plasmodium Chabaudi: Effect of Antimalarial Drugs on Gametocytogenesis. Exp. Parasitol. 93 (1), 45-54. doi: 10.1006/expr.1999.4429

Buckling, A., Ranford-Cartwright, L. C., Miles, A., and Read, A. F. (1999b). Chloroquine Increases Plasmodium Falciparum Gametocytogenesis In Vitro. Parasitology 118 (Pt 4), 339-346. doi: 10.1017/S0031182099003960

Buckling, A. G., Taylor, L. H., Carlton, J. M., and Read, A. F. (1997). Adaptive Changes in Plasmodium Transmission Strategies Following Chloroquine Chemotherapy. Proc. Biol. Sci. 264 (1381), 553-559. doi: 10.1098/rspb.1997. 0079

Carter, L. M., Kafsack, B. F. C., Llinás, M., Mideo, N., Pollitt, L. C., and Reece, S. E. (2013). Stress and Sex in Malaria Parasites: Why Does Commitment Vary? Evol. Med. Public Health 2013 (1), 135-147. doi: 10.1093/emph/eot011 
Cerletti, C., Bonati, M., del Maschio, A., Galletti, F., Dejana, E., Tognoni, G., et al. (1984). Plasma Levels of Salicylate and Aspirin in Healthy Volunteers: Relevance to Drug Interaction on Platelet Function. J. Lab. Clin. Med. 103 (6), 869-877.

Chaubey, S., Grover, M., and Tatu, U. (2014). Endoplasmic Reticulum Stress Triggers Gametocytogenesis in the Malaria Parasite. J. Biol. Chem. 289 (24), 16662-16674. doi: 10.1074/jbc.M114.551549

Chua, M. J., Arnold, M. S., Xu, W., Lancelot, J., Lamotte, S., Späth, G. F., et al. (2017). Effect of Clinically Approved HDAC Inhibitors on Plasmodium, Leishmania and Schistosoma Parasite Growth. Int. J. Parasitol. Drugs Drug Resist. 7 (1), 42-50. doi: 10.1016/j.ijpddr.2016.12.005

Chugh, M., Scheurer, C., Sax, S., Bilsland, E., van Schalkwyk, D. A., Wicht, K. J., et al. (2015). Identification and Deconvolution of Cross-Resistance Signals From Antimalarial Compounds Using Multidrug-Resistant Plasmodium Falciparum Strains. Antimicrob. Agents Chemother. 59 (2), 1110-1118. doi: 10.1128/AAC.03265-14

Coleman, B. I., Skillman, K. M., Jiang, R. H. Y., Childs, L. M., Altenhofen, L. M., Ganter, M., et al. (2014). A Plasmodium Falciparum Histone Deacetylase Regulates Antigenic Variation and Gametocyte Conversion. Cell Host Microbe 16 (2), 177-186. doi: 10.1016/j.chom.2014.06.014

Cotreau, M. M., Warren, S., Ryan, J. L., Fleckenstein, L., Vanapalli, S. R., Brown, K. R., et al. (2003). The Antiparasitic Moxidectin: Safety, Tolerability, and Pharmacokinetics in Humans. J. Clin. Pharmacol. 43 (10), 1108-1115. doi: 10.1177/0091270003257456

Croft, S. L. (2010). Antiprotozoal Agents. Antibiot. Chemother. 9, 406-426. doi: 10.1016/B978-0-7020-4064-1.00035-X

Delves, M. J., Straschil, U., Ruecker, A., Miguel-Blanco, C., Marques, S., Dufour, A. C., et al. (2016). Routine In Vitro Culture of P. Falciparum Gametocytes to Evaluate Novel Transmission-Blocking Interventions. Nat. Protoc. 11 (9), 1668-1680. doi: 10.1038/nprot.2016.096

Dixon, M. W., Peatey, C. L., Gardiner, D. L., and Trenholme, K. R. (2009). A Green Fluorescent Protein-Based Assay for Determining Gametocyte Production in Plasmodium Falciparum. Mol. Biochem. Parasitol. 163 (2), 123-126. doi: 10.1016/j.molbiopara.2008.10.004

Drakeley, C., Sutherland, C., Bousema, J. T., Sauerwein, R. W., and Targett, G. A. T. (2006). The Epidemiology of Plasmodium Falciparum Gametocytes: Weapons of Mass Dispersion. Trends Parasitol 22 (9), 424-430. doi: 10.1016/j.pt.2006.07.001

Dyer, M., and Day, K. P. (2003). Regulation of the Rate of Asexual Growth and Commitment to Sexual Development by Diffusible Factors From In Vitro Cultures of Plasmodium Falciparum. Am. J. Trop. Med. Hyg 68 (4), 403-409. doi: 10.4269/ajtmh.2003.68.403

Eckschlager, T., Plch, J., Stiborova, M., and Hrabeta, J. (2017). Histone Deacetylase Inhibitors as Anticancer Drugs. Int. J. Mol. Sci. 18 (7). doi: 10.3390/ ijms 18071414

Eksi, S., Morahan, B. J., Haile, Y., Furuya, T., Jiang, H., Ali, O., et al. (2012). Plasmodium Falciparum Gametocyte Development 1 (Pfgdv1) and Gametocytogenesis Early Gene Identification and Commitment to Sexual Development. PloS Pathog. 8 (10), e1002964. doi: 10.1371/journal.ppat.1002964

Ezzet, F., van Vugt, M., Nosten, F., Looareesuwan, S., and White, N. J. (2000). Pharmacokinetics and Pharmacodynamics of Lumefantrine (Benflumetol) in Acute Falciparum Malaria. Antimicrob. Agents Chemother. 44 (3), 697-704. doi: 10.1128/AAC.44.3.697-704.2000

Filarsky, M., Fraschka, S. A., Niederwieser, I., Brancucci, N. M. B., Carrington, E., Carrió, E., et al. (2018). GDV1 Induces Sexual Commitment of Malaria Parasites by Antagonizing HP1-Dependent Gene Silencing. Science (New York NY) 359 (6381), 1259-1263. doi: 10.1126/science.aan6042

Fivelman, Q. L., McRobert, L., Sharp, S., Taylor, C. J., Saeed, M., Swales, C. A., et al. (2007). Improved Synchronous Production of Plasmodium Falciparum Gametocytes In Vitro. Mol. Biochem. Parasitol. 154 (1), 119-123. doi: 10.1016/j.molbiopara.2007.04.008

Flanagan, K. L., Buckley-Sharp, M., Doherty, T., and Whitty, C. J. (2006). Quinine Levels Revisited: The Value of Routine Drug Level Monitoring for Those on Parenteral Therapy. Acta Trop. 97 (2), 233-237. doi: 10.1016/j.actatropica.2005.11.006

Flueck, C., Bartfai, R., Volz, J., Niederwieser, I., Salcedo-Amaya, A. M., Alako, B. T. F., et al. (2009). Plasmodium Falciparum Heterochromatin Protein 1 Marks Genomic Loci Linked to Phenotypic Variation of Exported Virulence Factors. PloS Pathog. 5 (9), e1000569. doi: 10.1371/journal.ppat.1000569
Foulds, G., Shepard, R. M., and Johnson, R. B. (1990). The Pharmacokinetics of Azithromycin in Human Serum and Tissues. J. Antimicrob. Chemother. 25 Suppl A, 73-82. doi: 10.1093/jac/25.suppl_A.73

Gibson, D. G., Young, L., Chuang, R.-Y., Venter, J. C., Hutchison, C. A., and Smith, H. O. (2009). Enzymatic Assembly of DNA Molecules Up to Several Hundred Kilobases. Nat. Methods 6 (5), 343-345. doi: 10.1038/nmeth.1318

Gutman, J., Green, M., Durand, S., Rojas, O. V., Ganguly, B., Quezada, W. M., et al. (2009). Mefloquine Pharmacokinetics and Mefloquine-Artesunate Effectiveness in Peruvian Patients With Uncomplicated Plasmodium Falciparum Malaria. Malar. J. 8, 58. doi: 10.1186/1475-2875-8-58

Hawking, F., Wilson, M. E., and Gammage, K. (1971). Evidence for Cyclic Development and Short-Lived Maturity in the Gametocytes of Plasmodium Falciparum. Trans. R Soc. Trop. Med. Hyg. 65 (5), 549-559. doi: 10.1016/00359203(71)90036-8

Hess, C., Unger, M., Madea, B., Stratmann, B., and Tschoepe, D. (2018). Range of Therapeutic Metformin Concentrations in Clinical Blood Samples and Comparison to a Forensic Case With Death Due to Lactic Acidosis. Forensic Sci. Int. 286, 106-112. doi: 10.1016/j.forsciint.2018.03.003

Hogh, B., Gamage-Mendis, A., Butcher, G. A., Thompson, R., Begtrup, K., Mendis, C., et al. (1998). The Differing Impact of Chloroquine and Pyrimethamine/ Sulfadoxine Upon the Infectivity of Malaria Species to the Mosquito Vector. Am. J. Trop. Med. Hyg. 58 (2), 176-182. doi: 10.4269/ajtmh.1998.58.176

Hoglund, R. M., Workman, L., Edstein, M. D., Thanh, N. X., Quang, N. N., Zongo, I., et al. (2017). Population Pharmacokinetic Properties of Piperaquine in Falciparum Malaria: An Individual Participant Data Meta-Analysis. PloS Med. 14 (1), e1002212. doi: 10.1371/journal.pmed.1002212

Ippolito, M. M., Johnson, J., Mullin, C., Mallow, C., Morgan, N., Wallender, E., et al. (2017). The Relative Effects of Artemether-Lumefantrine and NonArtemisinin Antimalarials on Gametocyte Carriage and Transmission of Plasmodium Falciparum: A Systematic Review and Meta-Analysis. Clin. Infect. Dis. 65 (3), 486-494. doi: 10.1093/cid/cix336

Jensen, J. B., and Trager, W. (1978). Plasmodium Falciparum in Culture: Establishment of Additional Strains. Am. J. Trop. Med. Hyg 27 (4), 743-746. doi: 10.4269/ajtmh.1978.27.743

Jittamala, P., Pukrittayakamee, S., Ashley, E. A., Nosten, F., Hanboonkunupakarn, B., Lee, S. J., et al. (2015). Pharmacokinetic Interactions Between Primaquine and Pyronaridine-Artesunate in Healthy Adult Thai Subjects. Antimicrob. Agents Chemother. 59 (1), 505-513. doi: 10.1128/AAC.03829-14

Josling, G. A., Russell, T. J., Venezia, J., Orchard, L., van Biljon, R., Painter, H. J., et al. (2020). Dissecting the Role of Pfap2-G in Malaria Gametocytogenesis. Nat. Commun. 11 (1), 1503. doi: 10.1038/s41467-020-15026-0

Josling, G. A., Williamson, K. C., and Llinás, M. (2018). Regulation of Sexual Commitment and Gametocytogenesis in Malaria Parasites. Annu. Rev. Microbiol. 72, 501-519. doi: 10.1146/annurev-micro-090817-062712

Kafsack, B. F., Rovira-Graells, N., Clark, T. G., Bancells, C., Crowley, V. M., Campino, S. G., et al. (2014). A Transcriptional Switch Underlies Commitment to Sexual Development in Malaria Parasites. Nature 507 (7491), 248-252.

Karbwang, J., Looareesuwan, S., Phillips, R. E., Wattanagoon, Y., Molyneux, M. E., Nagachinta, B., et al. (1987). Plasma and Whole Blood Mefloquine Concentrations During Treatment of Chloroquine-Resistant Falciparum Malaria With the Combination Mefloquine-Sulphadoxine-Pyrimethamine. Br. J. Clin. Pharmacol. 23 (4), 477-481. doi: 10.1111/j.1365-2125.1987. tb03079.x

Kim, Y. L., Im, Y. J., Ha, N. C., and Im, D. S. (2007). Albumin Inhibits Cytotoxic Activity of Lysophosphatidylcholine by Direct Binding. Prostaglandins other Lipid Mediators 83 (1-2), 130-138. doi: 10.1016/j.prostaglandins.2006.10.006

Kumar, M., Skillman, K., and Duraisingh, M. T. (2021). Linking Nutrient Sensing and Gene Expression in Plasmodium Falciparum Blood-Stage Parasites. Mol. Microbiol. 115 (5), 891-900. doi: 10.1111/mmi.14652

Lacal, J. C., Zimmerman, T., and Campos, J. M. (2021). Choline Kinase: An Unexpected Journey for a Precision Medicine Strategy in Human Diseases. Pharmaceutics 13 (6). doi: 10.3390/pharmaceutics13060788

Lambros, C., and Vanderberg, J. P. (1979). Synchronization of Plasmodium Falciparum Erythrocytic Stages in Culture. J. Parasitol. 65 (3), 418-420. doi: $10.2307 / 3280287$

Lewis, M. D., Fortes Francisco, A., Taylor, M. C., Burrell-Saward, H., McLatchie, A. P., Miles, M. A., et al. (2014). Bioluminescence Imaging of Chronic Trypanosoma Cruzi Infections Reveals Tissue-Specific Parasite Dynamics 
and Heart Disease in the Absence of Locally Persistent Infection. Cell Microbiol. 16 (9), 1285-1300. doi: 10.1111/cmi.12297

Liu, Z., Chen, O., Wall, J. B. J., Zheng, M., Zhou, Y., Wang, L., et al. (2017). Systematic Comparison of 2A Peptides for Cloning Multi-Genes in a Polycistronic Vector. Sci. Rep. 7 (1), 2193. doi: 10.1038/s41598-017-02460-2

Llorà-Batlle, O., Michel-Todó, L., Witmer, K., Toda, H., Fernández-Becerra, C., Baum, J., et al. (2020). Conditional Expression of Pfap2-G for Controlled Massive Sexual Conversion in Plasmodium Falciparum. Sci. Adv. 6 (24), eaaz5057. doi: 10.1126/sciadv.aaz5057

Lopez-Rubio, J. J., Mancio-Silva, L., and Scherf, A. (2009). Genome-Wide Analysis of Heterochromatin Associates Clonally Variant Gene Regulation With Perinuclear Repressive Centers in Malaria Parasites. Cell Host Microbe 5 (2), 179-190. doi: 10.1016/j.chom.2008.12.012

Mantel, P. Y., Hoang, A. N., Goldowitz, I., Potashnikova, D., Hamza, B., Vorobjev, I., et al. (2013). Malaria-Infected Erythrocyte-Derived Microvesicles Mediate Cellular Communication Within the Parasite Population and With the Host Immune System. Cell Host Microbe 13 (5), 521-534. doi: 10.1016/j.chom. 2013.04.009

Maswoswe, S. M., Peters, W., and Warhurst, D. C. (1985). Corticosteroid Stimulation of the Growth of Plasmodium Falciparum Gametocytes In Vitro. Ann. Trop. Med. Parasitol. 79 (6), 607-616. doi: 10.1080/00034983. 1985.11811968

Mehlisch, D. R., Aspley, S., Daniels, S. E., Southerden, K. A., and Christensen, K. S. (2010). A Single-Tablet Fixed-Dose Combination of Racemic Ibuprofen/ Paracetamol in the Management of Moderate to Severe Postoperative Dental Pain in Adult and Adolescent Patients: A Multicenter, Two-Stage, Randomized, Double-Blind, Parallel-Group, Placebo-Controlled, Factorial Study. Clin. Ther. 32 (6), 1033-1049. doi: 10.1016/j.clinthera.2010.06.002

Mello, A., Vieira, M., Sena, L. W. P., Paixão, T. P. D., Pinto, A. C. G., Grisólia, D. P. A., et al. (2018). Levels of Primaquine and Carboxyprimaquine in Patients With Malaria Vivax From the Brazilian Amazon Basin. Rev. Inst Med. Trop. Sao Paulo 60, e66. doi: 10.1590/s1678-9946201860066

Méndez, F., Herrera, S., Murrain, B., Gutiérrez, A., Moreno, L. A., Manzano, M., et al. (2007). Selection of Antifolate-Resistant Plasmodium Falciparum by Sulfadoxine-Pyrimethamine Treatment and Infectivity to Anopheles Mosquitoes. Am. J. Trop. Med. Hyg. 77 (3), 438-443. doi: 10.4269/ajtmh. 2007.77.438

Miyatake, S., Ichiyama, H., Kondo, E., and Yasuda, K. (2009). Randomized Clinical Comparisons of Diclofenac Concentration in the Soft Tissues and Blood Plasma Between Topical and Oral Applications. Br. J. Clin. Pharmacol. 67 (1), 125-129. doi: 10.1111/j.1365-2125.2008.03333.x

Mockenhaupt, F. P., May, J., Bergqvist, Y., Ademowo, O. G., Olumese, P. E., Falusi, A. G., et al. (2000). Concentrations of Chloroquine and Malaria Parasites in Blood in Nigerian Children. Antimicrob. Agents Chemother. 44 (4), 835-839. doi: 10.1128/AAC.44.4.835-839.2000

Neveu, G., Beri, D., and Kafsack, B. F. (2020). Metabolic Regulation of Sexual Commitment in Plasmodium Falciparum. Curr. Opin. Microbiol. 58, 93-98. doi: 10.1016/j.mib.2020.09.004

Newton, P. N., Chaulet, J. F., Brockman, A., Chierakul, W., Dondorp, A., Ruangveerayuth, R., et al. (2005). Pharmacokinetics of Oral Doxycycline During Combination Treatment of Severe Falciparum Malaria. Antimicrob. Agents Chemother. 49 (4), 1622-1625. doi: 10.1128/AAC.49.4.1622-1625.2005

Ngotho, P., Soares, A. B., Hentzschel, F., Achcar, F., Bertuccini, L., and Marti, M. (2019). Revisiting Gametocyte Biology in Malaria Parasites. FEMS Microbiol. Rev. 43 (4), 401-414. doi: 10.1093/femsre/fuz010

Ntale, M., Obua, C., Mukonzo, J., Mahindi, M., Gustafsson, L. L., Beck, O., et al. (2009). Field-Adapted Sampling of Whole Blood to Determine the Levels of Amodiaquine and Its Metabolite in Children With Uncomplicated Malaria Treated With Amodiaquine Plus Artesunate Combination. Malar. J. 8, 52. doi: 10.1186/1475-2875-8-52

Okell, L. C., Drakeley, C. J., Ghani, A. C., Bousema, T., and Sutherland, C. J. (2008). Reduction of Transmission From Malaria Patients by Artemisinin Combination Therapies: A Pooled Analysis of Six Randomized Trials. Malar. J. 7 (1), 125. doi: 10.1186/1475-2875-7-125

Olliaro, P., Delgado-Romero, P., and Keiser, J. (2014). The Little We Know About the Pharmacokinetics and Pharmacodynamics of Praziquantel (Racemate and R-Enantiomer). J. Antimicrob. Chemother. 69 (4), 863-870. doi: 10.1093/jac/ dkt491
Peatey, C. L., Leroy, D., Gardiner, D. L., and Trenholme, K. R. (2012). AntiMalarial Drugs: How Effective Are They Against Plasmodium Falciparum Gametocytes? Malar. J. 11, 34. doi: 10.1186/1475-2875-11-34

Peatey, C. L., Skinner-Adams, T. S., Dixon, M. W., McCarthy, J. S., Gardiner, D. L., and Trenholme, K. R. (2009). Effect of Antimalarial Drugs on Plasmodium Falciparum Gametocytes. J. Infect. Dis. 200 (10), 1518-1521. doi: 10.1086/ 644645

Plouffe, D. M., Wree, M., Du, A. Y., Meister, S., Li, F., Patra, K., et al. (2016). HighThroughput Assay and Discovery of Small Molecules That Interrupt Malaria Transmission. Cell Host Microbe 19 (1), 114-126. doi: 10.1016/j.chom. 2015.12.001

Portugaliza, H. P., Llorà-Batlle, O., Rosanas-Urgell, A., and Cortés, A. (2019). Reporter Lines Based on the Gexp02 Promoter Enable Early Quantification of Sexual Conversion Rates in the Malaria Parasite Plasmodium Falciparum. Sci. Rep. 9 (1), 14595. doi: 10.1038/s41598-019-50768-y

Portugaliza, H. P., Miyazaki, S., Geurten, F. J. A., Pell, C., Rosanas-Urgell, A., Janse, C. J., et al. (2020). Artemisinin Exposure at the Ring or Trophozoite Stage Impacts Plasmodium Falciparum Sexual Conversion Differently. eLife 9, e60058. doi: 10.7554/eLife.60058.sa2

Price, R., Nosten, F., Simpson, J. A., Luxemburger, C., Phaipun, L., ter Kuile, F., et al. (1999). Risk Factors for Gametocyte Carriage in Uncomplicated Falciparum Malaria. Am. J. Trop. Med. Hyg. 60 (6), 1019-1023. doi: 10.4269/ ajtmh.1999.60.1019

Puta, C., and Manyando, C. (1997). Enhanced Gametocyte Production in Fansidar-Treated Plasmodium Falciparum Malaria Patients: Implications for Malaria Transmission Control Programmes. Trop. Med. Int. Health 2 (3), $227-$ 229. doi: 10.1046/j.1365-3156.1997.d01-267.x

Reece, S. E., Ali, E., Schneider, P., and Babiker, H. A. (2010). Stress, Drugs and the Evolution of Reproductive Restraint in Malaria Parasites. Proc. Biol. Sci. 277 (1697), 3123-3129. doi: 10.1098/rspb.2010.0564

Regev-Rudzki, N., Wilson, D. W., Carvalho, T. G., Sisquella, X., Coleman, B. M., Rug, M., et al. (2013). Cell-Cell Communication Between Malaria-Infected Red Blood Cells via Exosome-Like Vesicles. Cell 153 (5), 1120-1133. doi: 10.1016/ j.cell.2013.04.029

Salcedo-Amaya, A. M., van Driel, M. A., Alako, B. T., Trelle, M. B., van den Elzen, A. M., Cohen, A. M., et al. (2009). Dynamic Histone H3 Epigenome Marking During the Intraerythrocytic Cycle of Plasmodium Falciparum. Proc. Natl. Acad. Sci. U. S. A. 106 (24), 9655-9660. doi: 10.1073/pnas. 0902515106

Saljoughian, M. (2016). Acetaminophen Intoxication: A Critical-Care Emergency. US Pharm. 41 (12), 38-41.

Saunders, D. L., Vanachayangkul, P., and Lon, C. (2014). DihydroartemisininPiperaquine Failure in Cambodia. N. Engl. J. Med. 371 (5), 484-485. doi: 10.1056/NEJMc1403007

Schulz, J. D., Neodo, A., Coulibaly, J. T., and Keiser, J. (2019). Pharmacokinetics of Albendazole, Albendazole Sulfoxide, and Albendazole Sulfone Determined From Plasma, Blood, Dried-Blood Spots, and Mitra Samples of HookwormInfected Adolescents. Antimicrob. Agents Chemother. 63 (4). doi: 10.1128/ AAC. $02489-18$

Sinha, A., Hughes, K. R., Modrzynska, K. K., Otto, T. D., Pfander, C., Dickens, N. J., et al. (2014). A Cascade of DNA-Binding Proteins for Sexual Commitment and Development in Plasmodium. Nature 507 (7491), 253-257. doi: 10.1038/ nature 12970

Taylor, L. H., and Read, A. F. (1997). Why So Few Transmission Stages? Reproductive Restraint by Malaria Parasites. Parasitol. Today 13 (4), 135140. doi: 10.1016/S0169-4758(97)89810-9

Trenque, T., Simon, N., Villena, I., Chemla, C., Quereux, C., Leroux, B., et al. (2004). Population Pharmacokinetics of Pyrimethamine and Sulfadoxine in Children With Congenital Toxoplasmosis. Br. J. Clin. Pharmacol. 57 (6), $735-$ 741. doi: 10.1111/j.1365-2125.2004.02077.x

Usui, M., Prajapati, S. K., Ayanful-Torgby, R., Acquah, F. K., Cudjoe, E., Kakaney, C., et al. (2019). Plasmodium Falciparum Sexual Differentiation in Malaria Patients Is Associated With Host Factors and GDV1-Dependent Genes. Nat. Commun. 10 (1), 2140. doi: 10.1038/s41467-019-10172-6

von Nicolai, H., Brickl, R., Eschey, H., Greischel, A., Heinzel, G., König, E., et al. (1997). Duration of Action and Pharmacokinetics of the Oral Antidiabetic Drug Gliquidone in Patients With Non-Insulin-Dependent (Type 2) Diabetes Mellitus. Arzneimittelforschung 47 (3), 247-252. 
Wattanagoon, Y., Taylor, R. B., Moody, R. R., Ochekpe, N. A., Looareesuwan, S., and White, N. J. (1987). Single Dose Pharmacokinetics of Proguanil and Its Metabolites in Healthy Subjects. Br. J. Clin. Pharmacol. 24 (6), 775-780. doi: 10.1111/j.1365-2125.1987.tb03245.x

White, N. J. (2008). The Role of Anti-Malarial Drugs in Eliminating Malaria. Malaria J. 7 (1), S8. doi: 10.1186/1475-2875-7-S1-S8

WHO. (2020). World Malaria Report 2020 (Geneva, Switzerland: WHO).

Williams, J. L. (1999). Stimulation of Plasmodium Falciparum Gametocytogenesis by Conditioned Medium From Parasite Cultures. Am. J. Trop. Med. Hyg 60 (1), 7-13. doi: 10.4269/ajtmh.1999.60.7

Wong, W., Bai, X. C., Sleebs, B. E., Triglia, T., Brown, A., Thompson, J. K., et al. (2017). Mefloquine Targets the Plasmodium Falciparum 80S Ribosome to Inhibit Protein Synthesis. Nat. Microbiol. 2, 17031. doi: 10.1038/nmicrobiol.2017.31

Yang, L., Panetta, J. C., Cai, X., Yang, W., Pei, D., Cheng, C., et al. (2008). Asparaginase may Influence Dexamethasone Pharmacokinetics in Acute Lymphoblastic Leukemia. J. Clin. Oncol. 26 (12), 1932-1939. doi: 10.1200/JCO.2007.13.8404
Conflict of Interest: The authors declare that the research was conducted in the absence of any commercial or financial relationships that could be construed as a potential conflict of interest.

Publisher's Note: All claims expressed in this article are solely those of the authors and do not necessarily represent those of their affiliated organizations, or those of the publisher, the editors and the reviewers. Any product that may be evaluated in this article, or claim that may be made by its manufacturer, is not guaranteed or endorsed by the publisher.

Copyright (๑) 2022 Thommen, Passecker, Buser, Hitz, Voss and Brancucci. This is an open-access article distributed under the terms of the Creative Commons Attribution License (CC BY). The use, distribution or reproduction in other forums is permitted, provided the original author(s) and the copyright owner(s) are credited and that the original publication in this journal is cited, in accordance with accepted academic practice. No use, distribution or reproduction is permitted which does not comply with these terms. 